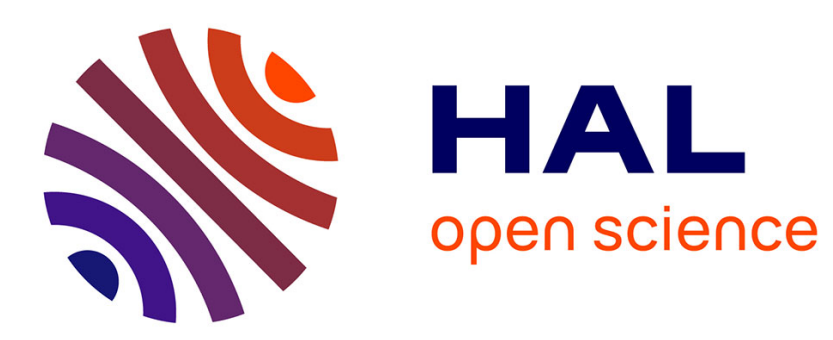

\title{
Turbulent bubbly flow in pipe under gravity and microgravity conditions
}

Catherine Colin, Jean Fabre, Arjan Kamp

\section{To cite this version:}

Catherine Colin, Jean Fabre, Arjan Kamp. Turbulent bubbly flow in pipe under gravity and microgravity conditions. Journal of Fluid Mechanics, 2012, vol. 711, pp. 469-515. 10.1017/jfm.2012.401 . hal-00875768

\section{HAL Id: hal-00875768 \\ https://hal.science/hal-00875768}

Submitted on 22 Oct 2013

HAL is a multi-disciplinary open access archive for the deposit and dissemination of scientific research documents, whether they are published or not. The documents may come from teaching and research institutions in France or abroad, or from public or private research centers.
L'archive ouverte pluridisciplinaire HAL, est destinée au dépôt et à la diffusion de documents scientifiques de niveau recherche, publiés ou non, émanant des établissements d'enseignement et de recherche français ou étrangers, des laboratoires publics ou privés. 


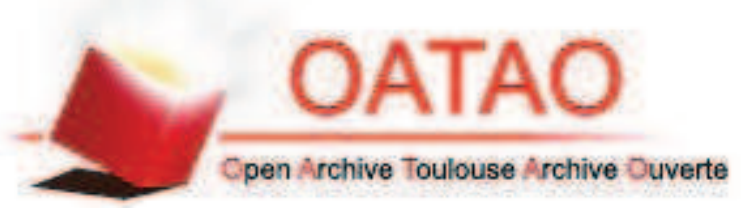

\section{Open Archive TOULOUSE Archive Ouverte (OATAO)}

OATAO is an open access repository that collects the work of Toulouse researchers and makes it freely available over the web where possible.

This is an author-deposited version published in : http://oatao.univ-toulouse.fr/ Eprints ID : 9788

To link to this article :DOI $: 10.1017 / \mathrm{jm} .2012 .401$

URL : http://dx.doi.org/10.1017/jfm.2012.401

To cite this version Colin, Catherine and Fabre, Jean and Kamp, Arjan

Turbulent bubbly flow in pipe under gravity and microgravity conditions.

(2012) Journal of Fluid Mechanics, vol. 711 . pp. 469-515. ISSN 0022-1120

Any correspondance concerning this service should be sent to the repository administrator: staff-oatao@,1istes-diff.inp-toulouse.fr 


\title{
Turbulent bubbly flow in pipe under gravity and microgravity conditions
}

\author{
Catherine Colin ${ }^{1}$, Jean Fabre ${ }^{1} \dagger$ and Arjan Kamp ${ }^{2}$ \\ ${ }^{1}$ Institut de Mécanique des Fluides, Institut National Polytechnique de Toulouse, \\ Allée du Prof. Camille Soula, 31400 Toulouse, France \\ ${ }^{2}$ Centre Scientifique et Technique Jean-Féger, TOTAL, Avenue Larribau, 64018 Pau, France
}

Experiments on vertical turbulent flow with millimetric bubbles, under three gravity conditions, upward, downward and microgravity flows $(1 g,-1 g$ and $0 g$ ), have been performed to understand the influence of gravity upon the flow structure and the phase distribution. The mean and fluctuating phase velocities, shear stress, turbulence production, gas fraction and bubble size have been measured or determined. The results for $0 g$ flow obtained during parabolic flights are taken as reference for buoyant $1 g$ and $-1 g$ flows. Three buoyancy numbers are introduced to understand and quantify the effects of gravity with respect to friction. We show that the kinematic structure of the liquid is similar to single-phase flow for $0 g$ flow whereas it deviates in $1 g$ and $-1 g$ buoyant flows. The present results confirm the existence of a two-layer structure for buoyant flows with a nearly homogeneous core and a wall layer similar to the singlephase inertial layer whose thickness seems to result from a friction-gravity balance. The distributions of phase velocity, shear stress and turbulence are discussed in the light of various existing physical models. This leads to a dimensionless correlation that quantifies the wall shear stress increase due to buoyancy. The turbulent dispersion, the lift and the nonlinear effects of added mass are taken into account in a simplified model for the phase distribution. Its analytical solution gives a qualitative description of the gas fraction distribution in the wall layer.

Key words: bubble dynamics, gas/liquid flow, turbulent flow

\section{Introduction}

During the past thirty years, bubbly flow in pipe has been extensively investigated for several reasons. It is the simplest fully developed two-phase flow and despite this simplicity it addresses some of the most important issues related to the bubble dynamics in turbulent flow. Besides, it has several practical applications in thermal, nuclear and chemical industries. As a consequence a large amount of experimental results have been accumulated thanks to various measurement methods. These results concern the radial distributions of phase fraction, bubble velocity, bubble size, liquid velocity and turbulence.

Most of these studies were focused on vertical upward flow with particular attention paid to the radial distribution of bubbles and to the turbulence that they induce in the liquid. Local gas fraction, bubble velocity and bubble size were measured by a 
resistive probe (Herringe \& Davis 1976; van der Welle 1985; Liu \& Bankoff 1993a), by an optical probe (Grossetête 1995; Kamp 1996; Shawkat, Ching \& Shoukri 2008) and by a wire mesh sensor (Prasser, Krepper \& Lucas 2002). Different gas fraction distributions were observed: a void peak consisting of a bubble layer near the pipe wall or a void core with a convex profile. Intermediate shapes were also observed with a peak in the gas fraction profiles near the wall and a secondary smaller peak farther from the wall. The radial migration of the bubbles was shown to be strongly dependent on their size (Zun 1988; Zun et al. 1993; Liu 1993, 1998). Bubbles of $2-3 \mathrm{~mm}$ diameter move toward the pipe wall under the effect of the lift force whereas larger bubbles move toward the pipe centre due to the lift reversal (Tomiyama et al. 2002). The action of the pressure gradient in the radial direction due to the non-uniformity of the turbulence of the liquid phase (Drew \& Lahey 1982) and the turbulent dispersion by the turbulent eddies also play a significant role in the radial bubble distribution (Lopez de Bertodano, Lahey \& Jones 1994; Lucas, Krepper \& Prasser 2007; Shawkat et al. 2008).

In most of the experiments the velocity of the liquid phase was measured either by laser Doppler anemometry (Theofanous \& Sullivan 1982; Hosokawa \& Tomiyama 2004) or by a hot film (Wang et al. 1987; Liu \& Bankoff 1993a; Shawkat et al. 2008) or by electrochemical and conductivity techniques (Nakoryakov et al. 1981). Particle image velocimetry (Hasan, Schmidl \& Ortiz-Villafuerte 1998; Fujiwara, Minato \& Hishida 2004) and more recently molecular tagging velocimetry (Hosokawa \& Tomiyama 2009a) were also used to investigate the three-dimensional structure of turbulence and the budget of turbulence kinetic energy. One of the main findings is that the bubbles tend to flatten the velocity profile when a peak of gas fraction exists near the wall, whereas the opposite is observed when the gas fraction is maximal at the axis. Another major finding concerns the turbulence enhancement or suppression in comparison to single-phase flow (Serizawa, Kataoka \& Michiyoshi 1975; Michiyoshi \& Serizawa 1986; Wang et al. 1987) depending on the ratio of bubble-induced to shear-induced turbulence (Shawkat et al. 2008).

Fewer studies consider vertical downward flow. The experimental results concern the radial distribution of gas fraction (Wang 1985; Nakoryakov et al. 1996), bubble size and velocity (Hibiki et al. 2004), liquid velocity and turbulence (Kashinsky \& Randin 1999). In these experiments a migration of the bubbles toward the pipe centre was observed. The mean velocity of the liquid is smaller than in single-phase flow near the pipe centre, and so the velocity profiles are flatter. As in upward flow at low liquid flow rate, the turbulence level is higher than in single-phase flow, whereas it can be smaller for high liquid flow rate and low gas fraction.

There are even fewer studies on the local structure of bubbly flow at microgravity conditions. Examples of them are the experiments of Lahey \& Bonetto (1994) that mimic microgravity flow by using neutrally buoyant particles, those of Takamasa et al. (2003) and Hazaku, Takamasa \& Hibiki (2012) who investigated the behaviour of the dispersed phase in bubbly flow through the distribution of phase, interfacial area and mean velocity, and those of Kamp (1996) that will be extensively used in the present study because the data are the only ones that cover both the gas and the liquid phases. What makes two-phase flow, and especially bubbly flow, so specific is that it is extremely sensitive to gravity. A thorough and comprehensive review of the numerous experimental studies available today reveals that we have only a fragmentary knowledge of its effect. Moreover the corresponding data are usually incomplete and sometimes contradictory. Thanks to experimental results that were obtained under various gravity conditions (upward, downward and microgravity flow) in the same 


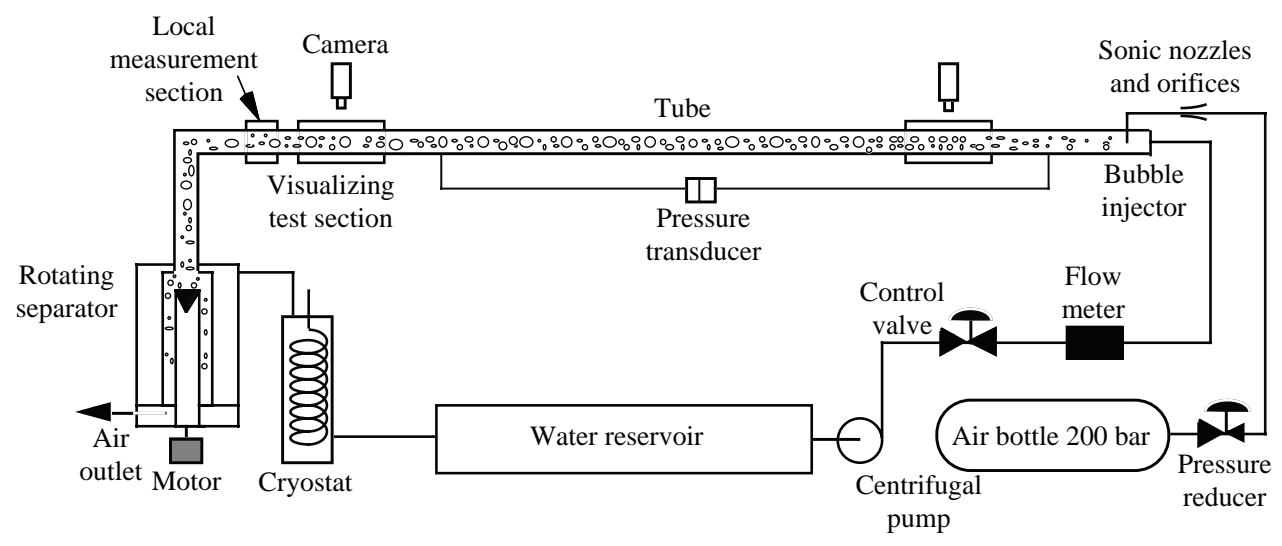

FIGURE 1. Experimental facility.

facility (Kamp 1996) and that were never published in their entirety, we can provide some possible answers to this important issue. This set of data is unique because it involves three different gravity conditions and in particular a microgravity condition that can be regarded as a reference case. In the absence of buoyancy, the bubble slip is suppressed and so are the lift force and the bubble-induced turbulence. The objective of the present study is therefore to quantify the contribution of buoyancy to flow dynamics and phase distribution.

Bubbly flow experiments were performed in a pipe of $4 \mathrm{~cm}$ diameter with bubbles of 2-4 mm diameter, comparable to the turbulence length scale. To highlight the role of gravity in the phase distribution and the turbulence in the liquid, the experiments were carried out with the same experimental set-up in microgravity, upward and downward flows. The experimental facility and the experimental conditions are presented in $\S 2$. The measurements techniques involving an optical probe for the characterization of the gas phase and a thermal probe for the measurement of the liquid velocity are described in $\S \S 3$ and 4 . The radial distributions of gas fraction, bubble diameter, and gas and liquid mean and fluctuating velocities are presented in $\S 5$. In $\S 6$, the relative contributions of friction and buoyancy to the kinematic flow structure and the phase distribution are demonstrated and correlated to the value of various buoyancy numbers that were introduced for this purpose.

\section{Experimental facility and operating conditions}

\subsection{Experimental facility}

The experimental facility (figure 1) was designed to perform ground-based experiments in upward and downward flows as well as in parabolic flight experiments at microgravity conditions. Apart from the mixer and the local instrumentation, this facility is the same as the one presented in Colin, Fabre \& Dukler (1991). The test section consisted of a transparent Plexiglas pipe of $40 \mathrm{~mm}$ inner diameter $D$ and $3.17 \mathrm{~m}$ length. Near the inlet and the outlet, the pipe was surrounded with parallelepiped-shaped boxes filled with water to reduce the image distortion of the flow visualizations. For the present experiments a specific mixer was designed. The water was introduced in the pipe axially and the air bubbles were injected through 24 hypodermic needles of $0.34 \mathrm{~mm}$ diameter, among which eight were uniformly 
located on a circle of $16 \mathrm{~mm}$ diameter and 16 on a circle of $32 \mathrm{~mm}$ diameter. This device provided a uniform bubble size distribution.

The microgravity experiments imposed some special constraints for the fluid management. Air was stored in a 201 tank under the initial pressure of 200 bars and supplied at a lower pressure through a sonic nozzle. The air flow rate was measured with one of the orifice plates covering the range of gas flow conditions. Water was stored in a 501 reservoir and circulated by a centrifugal pump providing $1201 \mathrm{~min}^{-1}$ under 1.5 bar pressure. Its flow rate was measured by an electromagnetic flowmeter. The temperature of the liquid was kept constant by a cooling system. As the volume consumed during the flights exceeded the volume of the reservoir it was necessary to circulate the water. A rotating gas-liquid separator was located at the outlet of the test section (Colin et al. 1991).

The test section was equipped with pressure taps for the determination of the pressure gradient and with a conductive probe for the volumetric gas fraction. This probe, located downstream of the local measurement test section (figure 1), consists of three pairs of electrodes, each electrode being a quarter of a cylinder flushed mounted at the pipe wall (Colin et al. 1991). The main electrode pair of $3 \mathrm{~cm}$ height is placed between two guard electrode pairs of $1 \mathrm{~cm}$ height. Taking into account the distance between each pair, the measurement volume can be viewed as a cylinder of height $D$ whose conductance, maximum when filled with water, decreases with increasing gas fraction. The probe is calibrated in bubbly flow by using quick-closing valves.

High-speed video pictures were also taken through the visualization sections with two high-speed video cameras (Kodak Ektapro EM) operating at 1000 frames s$^{-1}$. The bubble size and velocity distributions were obtained by image processing.

At $70 D$ from the mixer the test section was also equipped with a traversing mechanism controlled by a micrometre screw that allowed the radial displacement of a single hot-film probe or a dual fibre-optical probe. The uncertainty in the probe position was smaller than $0.05 \mathrm{~mm}$. The radial distributions of gas fraction, bubble velocity and bubble diameter were determined from the measurements performed with the dual optical probe. The liquid velocity in the axial direction was measured by hot-film anemometry. The local measurements required specific signal processing described in $\S \S 3$ and 4 .

\subsection{Flow conditions}

The liquid volumetric flux $j_{L}$ ranged between 0.27 and $1 \mathrm{~m} \mathrm{~s}^{-1}$ and the gas flux $j_{G}$ was smaller than $0.05 \mathrm{~m} \mathrm{~s}^{-1}$. The flow conditions are indicated in table 1 . For each liquid flux value we performed upward, downward and microgravity experiments. The first column contains the reference of each run. It consists of a letter and a number. The letter characterizes the gravity condition (S, U, D, M are for single-phase, upward, downward and microgravity flow respectively) and the number times $10^{4}$ is about the value of the Reynolds number as defined below. The table also specifies:

(i) the pressure gradient, $\mathrm{d} P / \mathrm{d} x$, determined from pressure transducers;

(ii) the volumetric gas fraction, $\langle\alpha\rangle_{m}$, measured by conductive probes;

(iii) the mean gas fraction, $\langle\alpha\rangle$, determined from a cross-sectional average of its local value $\alpha$, whenever it was measured;

(iv) the mean fluxes of gas and liquid, $\left\langle\alpha U_{G}\right\rangle$ and $\left\langle(1-\alpha) U_{L}\right\rangle$, whenever $\alpha$ was known; 


\begin{tabular}{|c|c|c|c|c|c|c|c|c|c|c|c|c|c|}
\hline Run & $\begin{array}{c}j_{L} \\
\left(\mathrm{~m} \mathrm{~s}^{-1}\right)\end{array}$ & $\begin{array}{c}j_{G} \\
\left(\mathrm{~m} \mathrm{~s}^{-1}\right)\end{array}$ & $\begin{array}{c}\langle\alpha\rangle_{m} \\
\%\end{array}$ & $\begin{array}{l}\left\langle\alpha_{L} U_{L}\right\rangle \\
\left(\mathrm{m} \mathrm{s}^{-1}\right)\end{array}$ & $\begin{array}{l}\left\langle\alpha U_{G}\right\rangle \\
\left(\mathrm{m} \mathrm{s}^{-1}\right)\end{array}$ & $\begin{array}{l}\langle\alpha\rangle \\
\%\end{array}$ & $\begin{array}{c}\mathrm{d} P / \mathrm{d} z \\
\left(\mathrm{~Pa} \mathrm{~m}^{-1}\right)\end{array}$ & $\begin{array}{c}4 \tau_{w} / D \\
\left(\mathrm{~Pa} \mathrm{~m}^{-1}\right)\end{array}$ & $\begin{array}{c}u_{*} \\
\left(\mathrm{~m} \mathrm{~s}^{-1}\right)\end{array}$ & $\begin{array}{c}u_{* 0} \\
\left(\mathrm{~m} \mathrm{~s}^{-1}\right)\end{array}$ & $\begin{array}{c}d \\
(\mathrm{~mm})\end{array}$ & $\underline{R i_{*}}$ & Re \\
\hline S1 & 0.27 & 0 & 0 & 0.26 & 0 & 0 & -29 & -29 & 0.017 & 0.017 & - & 0 & 10800 \\
\hline S3 & 0.77 & 0 & 0 & 0.74 & 0 & 0 & -160 & -160 & 0.040 & 0.041 & - & 0 & 30800 \\
\hline S4 & 1 & 0 & 0 & 1 & 0 & 0 & -281 & -281 & 0.053 & 0.052 & - & 0 & 40000 \\
\hline U1 & 0.27 & 0.023 & 3.3 & 0.24 & 0.021 & 4.3 & -9542 & -154 & 0.039 & 0.017 & 3.3 & 2.74 & 11720 \\
\hline U3 & 0.77 & 0.046 & 3.8 & 0.75 & 0.044 & 4.3 & -9669 & -281 & 0.053 & 0.043 & 3.5 & 1.50 & 32640 \\
\hline U4 & 1 & 0.023 & 1.8 & 0.95 & 0.022 & 1.9 & -9935 & -311 & 0.056 & 0.053 & 3.4 & 0.60 & 40920 \\
\hline $\mathrm{U} 4 \mathrm{a}$ & 1 & 0.034 & 2.2 & - & 0.035 & 2.7 & -9914 & -369 & 0.061 & 0.053 & 3.4 & 0.72 & 41360 \\
\hline D2a & 0.5 & 0.018 & 6.8 & - & - & 4.9 & +9068 & -261 & 0.051 & 0.030 & 2.5 & 1.84 & 20720 \\
\hline D2 & 0.5 & 0.033 & 11.3 & - & - & 8.5 & +8635 & -341 & 0.058 & 0.031 & 2.4 & 2.44 & 21320 \\
\hline D3a & 0.77 & 0.028 & 5.4 & - & - & 4.1 & +9106 & -302 & 0.055 & 0.043 & 3.1 & 1.33 & 31920 \\
\hline D3 & 0.77 & 0.053 & 9.5 & 0.75 & 0.053 & 7.5 & +8662 & -412 & 0.064 & 0.044 & 4.2 & 1.78 & 32920 \\
\hline D4 & 1 & 0.024 & 3.1 & 1.05 & 0.024 & 2.4 & +9225 & -350 & 0.059 & 0.053 & 3.1 & 0.67 & 40960 \\
\hline D4a & 1 & 0.041 & 5.6 & - & - & 4.2 & +9014 & -384 & 0.062 & 0.054 & 4.2 & 1.07 & 41640 \\
\hline M1 & 0.27 & 0.030 & 10.0 & 0.24 & 0.021 & 5.5 & -35 & -35 & 0.019 & 0.017 & 1.8 & 0 & 12000 \\
\hline M3 & 0.78 & 0.046 & 5.4 & 0.75 & 0.044 & 4.1 & -222 & -222 & 0.047 & 0.043 & 2.0 & 0 & 33040 \\
\hline M4 & 1 & 0.028 & 3.2 & 0.95 & 0.022 & 2.5 & -360 & -360 & 0.060 & 0.053 & 1.2 & 0 & 41120 \\
\hline
\end{tabular}


(v) the friction velocity $u_{*}=\left(\left|\tau_{w}\right| / \rho_{L}\right)^{1 / 2}$ determined from the mixture momentum balance, assuming the flow fully developed and neglecting the gas weight:

$$
\tau_{w} \approx \frac{D}{4}\left[-\frac{\mathrm{d} P}{\mathrm{~d} x}-\rho_{L} \gamma(1-\langle\alpha\rangle)\right],
$$

where $\tau_{w}$ is the wall shear stress, $\rho_{L}$ is the liquid density and $\gamma$ is the acceleration due to gravity, equal to $-g, g, 0$ in downward, upward and microgravity flow respectively;

(vi) the single-phase friction velocity $u_{* 0}$, calculated with the Colebrook formula for the liquid flow of the same mean velocity $\left\langle U_{L}\right\rangle=j_{L} /(1-\langle\alpha\rangle)$;

(vii) the mean bubble diameter $d$;

(viii) the inverse frictional Froude number that we noted $R i_{*}$ because it has the form of a Richardson number:

$$
F r_{*}^{-1}=R i_{*}=\frac{|\gamma|\langle\alpha\rangle D}{4 u_{*}^{2}} ;
$$

(ix) and the Reynolds number based on the mixture velocity and the liquid viscosity:

$$
R e=\frac{\left(j_{L}+j_{G}\right) D}{v} .
$$

Note that the couples $j_{L}$ and $\left\langle(1-\alpha) U_{L}\right\rangle, j_{G}$ and $\left\langle\alpha U_{G}\right\rangle,\langle\alpha\rangle$ and $\langle\alpha\rangle_{m}$ correspond to different determinations of the same quantity. Their comparison is useful to assess the measurement accuracy. The difference between $j_{L}$ and $\left\langle(1-\alpha) U_{L}\right\rangle$ remains smaller than $5 \%$ except for the cases $\mathrm{U} 1$ and $\mathrm{M} 1$ for which it reaches $11 \%$. The difference of $9 \%$ between $j_{G}$ and $\left\langle\alpha U_{G}\right\rangle$ also remains acceptable except for the cases M1 and M4 for which it reaches $21 \%$ and $30 \%$ respectively. Finally $\left(\langle\alpha\rangle-\langle\alpha\rangle_{m}\right)$ is less than 0.01 except for the cases D3, M3 for which it reaches 0.02 and for the case M1 for which it is 0.045 . Note that for this run, the gas fraction distribution is no longer axisymmetric because of the dominant effect of the residual gravity leading to bubble stratification, as explained in the next section.

The local measurements of gas fraction, bubble size and axial velocities of gas and liquid were performed at a distance $x$ from the mixer equal to $2.8 \mathrm{~m}(x / D=70)$. Some measurements were also performed near the mixer at $x / D=11$.

The results of the experiments performed in upward, downward and microgravity flows, supplementary data available at http://www.journals.cambridge.org/flm, are presented in $\S 5$.

\subsection{Microgravity conditions}

The microgravity conditions were obtained during parabolic flights aboard the Caravelle ZERO-G aircraft of the French space agency CNES. Each flight campaign was composed of three flights, each with 30 parabolas. During each parabola a microgravity period of $20 \mathrm{~s}$ was available with a residual level of gravity smaller than $2 \%$ of the Earth's gravity. The procedure that was followed in microgravity deserves some particular comments for at least two reasons. On the one hand, each period of microgravity conditions is extremely short so that a specific procedure must be used to ensure the convergence of the statistical averages. On the other hand, in parabolic flights there exist various effects, especially in two-phase flow, that may cause the apparent acceleration experienced by the fluids to depart from zero: thus special attention must be paid to ensure that these effects have a negligible influence on the flow. 
To obtain the convergence of the statistical averages, for both the turbulence and the signal of the optical probe, five parabolas were necessary for each probe location: this corresponded to a total averaging time of $\sim 75 \mathrm{~s}$. Thus during each flight only six different radial positions can be investigated for a given flow condition.

The effect of the residual level of gravity on the bubble motion was evaluated. Let us consider the frame moving with the aircraft, $\boldsymbol{e}_{x}, \boldsymbol{e}_{y}, \boldsymbol{e}_{z}$ being the unit vectors in the longitudinal, the lateral and the normal directions to the floor respectively. In our experiment the pipe axis is aligned with $\boldsymbol{e}_{x}$. The aircraft follows a parabolic trajectory turning around an axis of rotation with an angular speed $\boldsymbol{\Omega}=\Omega \boldsymbol{e}_{y}$ of $\sim 0.06 \mathrm{rad} \mathrm{s}^{-1}$. The accelerometers located at the axis of rotation measure the $g$-jitter, i.e. the residual acceleration experienced by the aircraft at this point. The total residual acceleration experienced by the facility consists of the sum of the acceleration of the non-inertial frame, i.e. the plane acceleration including the $g$-jitter, and the Coriolis acceleration. Since the experimental facility is located at a distance $\Delta x=4 \mathrm{~m}$ behind the rotation axis, the entrainment acceleration in the axial direction is $\left|\Omega \wedge \Omega \wedge\left(\Delta x \boldsymbol{e}_{x}\right)\right| \approx 0.002 g$. Therefore a fluid particle moving in the pipe along the longitudinal axis of the aircraft with a velocity $V$ of $1 \mathrm{~m} \mathrm{~s}^{-1}$ experiences a Coriolis acceleration $2 \Omega \wedge\left(V \boldsymbol{e}_{x}\right)$ of $-0.01 \mathrm{~g}$ in the direction perpendicular to the pipe axis $\boldsymbol{e}_{z}$. The $g$-jitter measured by the accelerometers ranged between $\pm 0.03 g$. The total residual acceleration $J_{z}$ in the $z$-direction can therefore reach $0.04 \mathrm{~g}$ and it can disturb the bubble motion. This residual acceleration leads to a hydrostatic pressure difference over the bubble diameter $d$ equal to $\rho_{L} J_{z} d$. This pressure difference has to be compared to the effect of the turbulence responsible for the radial migration of the bubbles. In microgravity, the turbulence is only produced by the wall shear stress $\rho_{L} u_{*}^{2}$. The ratio of these two forces $u_{*}^{2} /\left(J_{z} d\right)$ is theoretically infinite in $0 g$ conditions. Because of the residual gravity in the parabolic flight experiments, it remains finite. For the cases M3 and M4 it is greater than 3 and 7 respectively but for the case M1 it is only 0.4. For this last case, the $g$-jitter is expected to disturb the radial migration of the bubbles in the pipe: it explains why $\langle\alpha\rangle,\left\langle(1-\alpha) U_{L}\right\rangle$ and $\left\langle\alpha U_{G}\right\rangle$, that result from local measurements, differ for this case from their corresponding global quantities $\langle\alpha\rangle_{m}, j_{L}, j_{G}$.

\section{Measurements in the gas phase}

Resistive and fibre-optical probes have been widely used in gas-liquid flow for the measurement of gas fraction, bubble size and velocity. Single probes have been used to detect the presence of each phase from the conductivity or optical index contrast between the phases whereas dual probes have been used to determine the bubble velocities and their chord lengths pierced by the probe tip. From the probability distribution of the chord lengths, the distribution of the bubble diameters can be calculated with an assumption on the bubble shapes. The relationship between the chord length distribution and the bubble size distribution has been given in previous studies (Herringe \& Davis 1976; Clark \& Turton 1988). Converting the chord length distribution into bubble size distribution requires a great number of measured chord lengths to avoid cumulative errors. In a statistical simulation Clark \& Turton (1988) used five thousand chord lengths to obtain a sufficiently accurate result. In their simulation a chord length distribution was generated by a Monte Carlo method from a given triangular bubble size distribution. The backward transformation of the chord length distribution provided a size distribution in good agreement with the triangular size distribution. To reduce the number of chord 
lengths, Liu \& Clark (1995) proposed a method to determine the mean and standard deviation of the bubble size distribution from the mean and standard deviation of the measured chord length distribution. These authors also developed an analytical approach to transform the chord length distribution into a bubble size distribution. In their method the chord length and bubble size distributions were assumed to follow either a Gamma probability function or a Rayleigh probability function.

In the present study, a similar approach was used to transform the measured chord length distributions into bubble sizes distributions (Clark \& Turton 1988), assuming that the bubble size distributions follow a log-normal law (Kamp 1996). The bubble size distributions, determined from the measurements with a dual fibre-optical probe, were compared to the bubble size distributions obtained from image processing of high-speed video recordings. In our experiments, the two fibres were $3.8 \mathrm{~mm}$ apart. The tip diameter was $\sim 50 \mu \mathrm{m}$.

\subsection{Gas fraction and bubble velocity}

The bubble presence was detected from the fibre probes. When their signal is greater than a threshold value the characteristic function of the gas phase is set to unity or else to zero. The local gas fraction followed directly from its time average.

Two steps were necessary to determine the velocity of individual bubbles. In a first step a characteristic time of transit $t_{p}$ of the gas between the tips of the dual probe was determined from the cross-correlation function of the probe signals; $t_{p}$ corresponds to the time of the maximum correlation. In a second step, we searched for the transit time of individual bubbles within a guessed interval $\left[t_{\min }, t_{\max }\right]$ around $t_{p}$. If an interface is detected at the upstream tip and another at the downstream tip at a later time $\Delta t$ that belongs to $\left[t_{\min }, t_{\max }\right]$, then the two interfaces are deemed to belong to the same bubble. Thus its corresponding velocity $u_{B}$ was calculated, knowing the distance $l$ between the tips: $u_{B}=l / \Delta t$. Then, the residence time $\Delta t_{1}$ of the bubble at the first tip was converted to a corresponding chord length $y$ from the measured bubble velocity $y=u_{B} \Delta t_{1}$. The mean velocity of the gas phase $U_{G}$ and its variance $\overline{u_{G}^{2}}$ were obtained by averaging the bubble velocities weighted by the residence time:

$$
U_{G}=\sum_{i=1}^{N} \Delta t_{1 i} u_{B i} / \sum_{i=1}^{N} \Delta t_{1 i}, \quad \overline{u_{G}^{2}}=\sum_{i=1}^{N} \Delta t_{1 i}\left(u_{B i}-U_{G}\right)^{2} / \sum_{i=1}^{N} \Delta t_{1 i} .
$$

\subsection{Bubble size distributions}

From a great number of bubbles pierced by the two tips, the chord length distribution was obtained and the corresponding bubble size distribution was determined, using the method of Clark \& Turton (1988).

The bubbles were assumed to move parallel to the direction of the probe tips. For a bubble size ranging between 1 and $6 \mathrm{~mm}$, their shape in water is spherical in $0 g$ flow and ellipsoidal in $1 g$ flow with a major axis $d$ and a minor axis $E d$, where the aspect ratio $E=E(d)$ depends on the bubble diameter $(E=1$ in $0 g$ flow). The ellipsoidal bubbles moved mainly in a direction parallel to their minor axis (Ellingsen \& Risso 2001). In what follows, the minor axis of the bubbles is supposed to be aligned with the probe axis. From the chord length distribution $W(y)$ measured by the optical-fibre probe, the bubble diameter distribution $P_{s}(d)$ is calculated by a backward transformation detailed in the Appendix. 


\begin{tabular}{lcccccccr}
$r^{+}$ & $\begin{array}{c}u_{L}^{\prime} \\
\left(\mathrm{m} \mathrm{s}^{-1}\right)\end{array}$ & $\begin{array}{c}U_{L} \\
\left(\mathrm{~m} \mathrm{~s}^{-1}\right)\end{array}$ & $\begin{array}{c}l_{e} \\
(\mathrm{~m})\end{array}$ & $\begin{array}{c}\lambda \\
(\mathrm{m})\end{array}$ & $\begin{array}{c}\eta \\
(\mathrm{m})\end{array}$ & $\begin{array}{c}l_{s} / \lambda \\
-\end{array}$ & $\begin{array}{c}t_{\lambda} \\
(\mathrm{s})\end{array}$ & \multicolumn{1}{c}{$\begin{array}{c}1 / t_{\lambda} \\
\left(\mathrm{s}^{-1}\right)\end{array}$} \\
0 & 0.05 & 1.22 & $8 \times 10^{-3}$ & $1.6 \times 10^{-3}$ & $9 \times 10^{-5}$ & 0.78 & $1.3 \times 10^{-3}$ & 763 \\
0.9 & 0.10 & 0.85 & $8 \times 10^{-4}$ & $3.5 \times 10^{-4}$ & $3 \times 10^{-5}$ & 3.60 & $4.1 \times 10^{-4}$ & 2400
\end{tabular}

TABLE 2. Turbulence space and time scales estimated in single-phase flow for $R e=40000$.

\section{Measurements in the liquid phase}

\subsection{Hot-wire probe characteristics and calibration}

The longitudinal instant velocity component of the liquid phase was measured by hotwire anemometry. A boundary layer probe Dantec 55R15, with a wire perpendicular to the flow direction was connected to the Dantec Streamline anemometer operated by a microcomputer. The measurements were performed with a constant overheat ratio equal to 1.07 . The overheat ratio was automatically adjusted by the Streamline anemometer before each measurement to avoid errors due to the change in the liquid temperature. The probe was calibrated in single-phase flow. It was located on the pipe axis and the calibration curve was obtained by varying the liquid flow rate $Q_{L}$. The output voltage of the anemometer was related to the mean liquid velocity on the pipe axis $U_{0}$ calculated from the liquid flow rate and an exponent $n$, whose value was fitted from the data given in Schlichting (1968, p. 599):

$$
U_{0}=\frac{(2 n+1)(n+1)}{2 n^{2}} \frac{4 Q_{L}}{\pi D^{2}},
$$

with $n=4.09 R e^{0.0467}$. The probe calibration was performed before and after each experiment. The wire of the probe had a diameter of $0.07 \mathrm{~mm}$ and a sensitive length of $1.25 \mathrm{~mm}$. It is important to estimate the size of the turbulent scales of the flow with respect to the film size. The wire diameter $d_{f}$ and its length $l_{f}$ have to be smaller than the turbulent length scale. The time and length scales of the turbulence were estimated in single-phase flow. Three length scales were calculated: the integral length scale $l_{e}$, the Taylor microscale $\lambda$ and the Kolmogorov length scale $\eta$ :

$$
l_{e}=\kappa y, \quad \lambda=\left(\frac{15 v u_{L}^{\prime 2}}{\varepsilon}\right)^{1 / 2}, \quad \eta=\left(\frac{v^{3}}{\varepsilon}\right)^{1 / 4},
$$

with $y=D / 2-r$ the distance from the wall, $v$ the liquid kinematic viscosity, $u_{L}^{\prime}$ a turbulence velocity scale and $\varepsilon=u_{L}^{\prime 3} / l_{e}$ the dissipation rate of the turbulence kinetic energy estimated from the integral length scale $l_{e}$. For a Reynolds number of 40000 corresponding to the highest flow rate of our experiments these length scales were determined at two radial positions $r$ of the pipe: at the pipe axis $(r=0)$ and as close as possible to the wall $(r=1.8 \mathrm{~cm})$. The different values are reported in table 2 . In the central part of the flow, the sensitive length of the wire $l_{s}$, is smaller than the Taylor microscale whereas in the near-wall region $l_{s}$ becomes greater than $\lambda$. The turbulent fluctuations are thus partly filtered near the wall. Across the pipe the Kolmogorov length scale remains of the same order of magnitude as the film diameter. The time scale $t_{\lambda}$ is equal to the Taylor microscale divided by the local mean axial velocity (table 2). The frequency of the signal acquisition was chosen greater than $1 / t_{\lambda}$ to avoid turbulence filtering. 

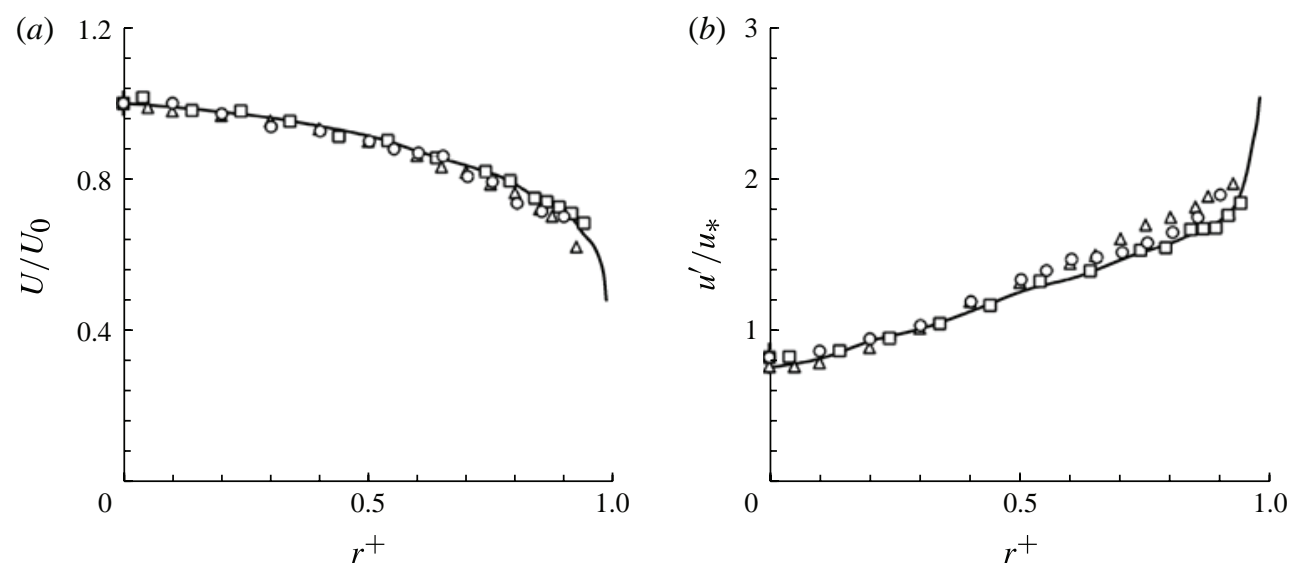

FigURE 2. Single-phase flow: $R e=11000(\bigcirc), 31000(\triangle), 50000(\square)$; Laufer (1954) (一). (a) Mean velocity; (b) r.m.s. velocity.

The mean axial velocity of the liquid $U_{L}$ and the turbulence fluctuation $\left(\overline{u_{L}^{2}}\right)^{1 / 2}$ denoted $u_{L}^{\prime}$ were measured at a distance $x=70 D$ from the pipe entrance for different Reynolds numbers $R e=j_{L} D / \nu$. The measurements were compared to Laufer (1954) at $R e=50000$. In figure 2 the mean axial velocity scaled by the axis velocity $U_{0}$ and the axial contribution of the turbulence energy scaled by the friction velocity $u_{*}$ are plotted versus the dimensionless radial coordinate $r^{+}=2 r / D$ : they are in good agreement with the Laufer results.

\subsection{Phase discrimination}

When the probe tip is piercing a bubble the output voltage of the anemometer drops, due to the weak cooling of the wire in air. The part of the signal corresponding to the bubble passage has to be suppressed before calculating the statistics of the liquid flow.

The passage time of a bubble of $1 \mathrm{~mm}$ diameter moving at $1 \mathrm{~m} \mathrm{~s}^{-1}$ is equal to $1 \mathrm{~ms}$. At least five points are required for the detection of the smallest and fastest bubbles: thus a sampling frequency $f_{s}$ of $5 \mathrm{kHz}$ was chosen for an accurate detection of the bubble passages from the output signal of the anemometer. The detection was performed using a classical algorithm that combines thresholds of both the slope and the level of the signal (Liu 1993; Farar, Samways \& Bruun 1995). The axial mean and root mean square (r.m.s.) velocity of the liquid phase, $U_{L}$ and $u_{L}^{\prime}$, were calculated. The uncertainty was $\sim 5 \%$ for the mean value and $10 \%$ for the r.m.s.

\section{Experimental results}

The analysis of the influence of the gravity upon the local structure of the flow requires experiments to be performed in the same experimental facility (same pipe diameter, same mixer and same fluid) with different levels of gravity. In this study the same two-phase flow loop was used for upward $(-1 g)$, microgravity $(0 g)$ and downward flow $(1 g)$. The experiments were carried out at three different classes of the Reynolds number $R e$ defined in (2.3): 10000,30000 and 40000 . It is expected that the shear-induced turbulence is nearly the same for flows belonging to the same class. The dimensionless number $R i_{*}$ expressing the ratio between the buoyancy and the wall friction and defined in (2.2) has been specified for each run in table 1. It is zero in 

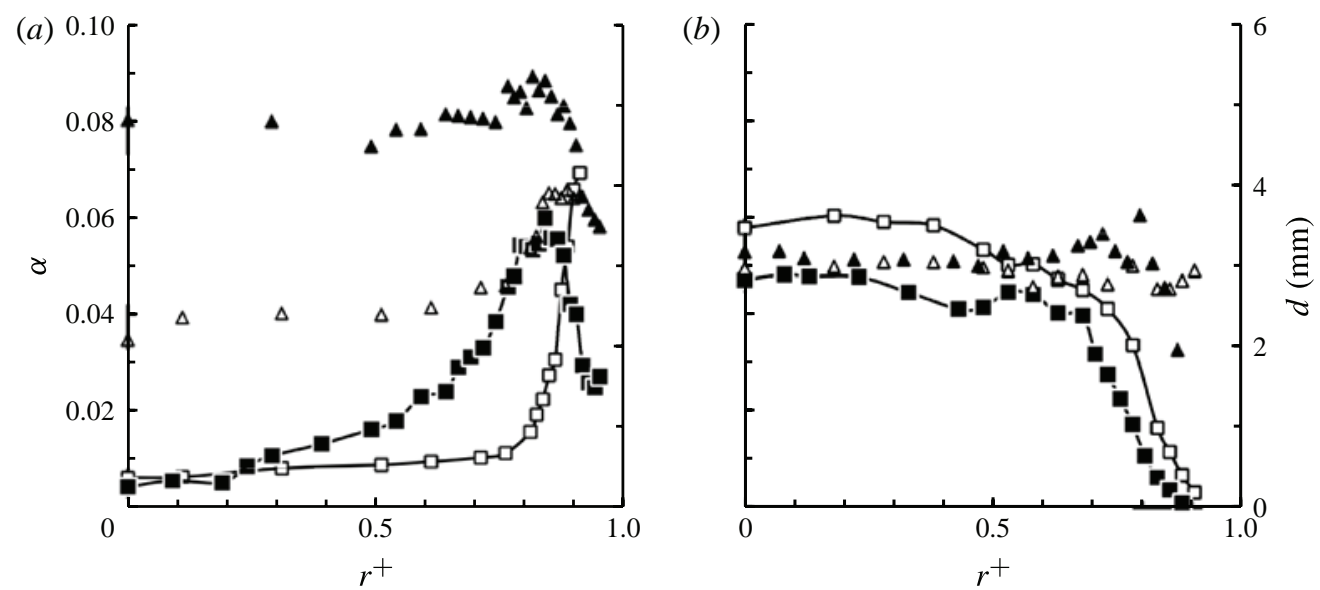

FIGURE 3. Evolution of gas fraction (squares) and bubble diameter (triangles) between $x / D=11$ (open symbols) and $x / D=70$ (solid symbols): $(a)$ upward flow; $(b)$ downward flow.

single-phase flow as well as at microgravity conditions where the only driving force is the pressure gradient. It would be nearly infinite in the case of bubbles rising freely in a vertical column: then the driving force would be the buoyancy. Between these two extreme cases there exist some intermediate situations like those investigated in the present study:

(i) The experiments for which $R i_{*}$ is smaller than 1 correspond to friction-dominated flows: S1, S2, S3, U4, D4, M1, M3, M4. The turbulence is mainly produced by the wall shear stress as is the case at microgravity conditions and the friction velocity $u_{*}$ is close to the single-phase value $u_{* 0}$

(ii) The large- $R i_{*}$ experiments correspond to gravity-dominated flows: U1, U3, D3. They are interesting to highlight the role of the bubble-induced turbulence.

\subsection{Flow development}

In two-phase vertical flow and more specifically in the bubbly regime, the flow is seldom fully developed. There are at least two reasons that prevent the flow reaching equilibrium:

(i) Coalescence or break-up that modifies the bubble diameter and thus the slip velocity. However far enough from the inlet, the mean bubble diameter should reach an equilibrium value.

(ii) Expansion or compression of the gas phase due to the pressure gradient. This effect may be significant in vertical flow because of the influence of the hydrostatic pressure gradient imposed by the weight of the liquid phase.

The influence of the bubble size and the developing length were clearly pointed out by Herringe \& Davis (1976), Serizawa \& Kataoka (1988) and Liu (1993) in upward flow. As the bubble size increases along the pipe due to bubble coalescence and gas expansion the gas fraction profile changes. The bubble diameter and the gas fraction were chosen for the present experiments to check the flow development. The results are shown in figure 3 in which the radial distributions of the gas fraction and the mean Sauter diameter are plotted for two different distances from the mixer. Bubbles 
of $\sim 2 \mathrm{~mm}$ diameter are generated through needles located at two radial positions: $r^{+}=0.4$ and $r^{+}=0.8$. The diameters are quite uniform, with an r.m.s. value of $10 \%$, and the bubbles are rapidly dispersed downstream of the mixer.

In upward flow, at only $11 D$ from the mixer (inlet), a void peaking is observed at a distance of $2 \mathrm{~mm}$ from the wall, corresponding to bubble diameter of $4 \mathrm{~mm}$. At the peak location a substantial increase in the bubble diameter is observed: this is consistent with the fact that the higher the gas fraction the greater the collision frequency and thus the bubble coalescence. At $x=70 \mathrm{D}$ (outlet), the peak in the gas fraction profile is weaker and located farther from the wall, because of the increase in bubble size. The bubble diameter ranges between 3 and $4 \mathrm{~mm}$. Some bubbles have migrated to the central part of the pipe. Zun (1988) also reported that in vertical upward flow, bubbles with a diameter smaller than $5 \mathrm{~mm}$ are driven towards the pipe wall, whereas bubbles with a diameter greater than $5 \mathrm{~mm}$ move toward the pipe centre. The experiments by Tomiyama et al. (2002) on transverse migration of single bubbles in simple shear flows clearly pointed out the change of the sign of the lift coefficient for a bubble diameter close to $5 \mathrm{~mm}$ in air-water systems.

In downward flow, the bubble diameter does not change significantly. It remains close to $3 \mathrm{~mm}$ and the radial distribution is rather uniform except close to the wall. The global gas fraction decreases slightly between $x=4 D$ (inlet) and $x=70 D$ (outlet): this is an effect of the hydrostatic pressure that increases the pressure and thus the gas density. The mean and r.m.s. gas velocities were also measured at $x=4 D$ and $x=70 D$. They do not evolve along the pipe, because the bubble diameter and thus the slip velocity does not change. In contrast to upward flow, the flow structure remains the same in downward flow. On the one hand, pressure and coalescence have opposite effects upon the development of bubble size, contrary to upward flow: coalescence tends to increase the mean diameter whereas pressure tends to decrease it whenever the hydrostatic pressure gradient is greater than the frictional pressure gradient. On the other hand, the rate of coalescence seems to be smaller in downward than in upward flow since near the outlet the mean bubble diameter reaches $5 \mathrm{~mm}$ in upward flow and $3 \mathrm{~mm}$ in downward flow. According to the model developed by Kamp et al. (2001), the coalescence rate is the product of the collision frequency and the coalescence probability. The collision frequency is proportional to the square of the gas fraction and to the bubble relative velocity. The coalescence probability is an exponential decreasing function of the r.m.s. of a Weber number $W e=\rho_{L}(\delta U)^{2} d / \sigma$, where $\delta U$ is the local instant relative velocity of two bubbles. The coalescence rate is thus proportional to $\alpha^{2} \delta U \exp \left[-\delta U\left(\rho_{L} d / \sigma\right)^{1 / 2}\right]$. Since the gas fraction in upward flow in the outer half of the pipe $\left(r^{+}>0.7\right)$ is comparable to that in downward flow in the inner half of the pipe $\left(r^{+}<0.7\right)$, the main difference should be attributed to $\delta U$. Indeed $\delta U$ is proportional to the turbulent velocity scale $u_{L}^{\prime}$, which is equal to $0.1 \mathrm{~m} \mathrm{~s}^{-1}$ in upward flow $\left(r^{+}>0.7\right)$ and $0.05 \mathrm{~m} \mathrm{~s}^{-1}$ in downward flow $\left(r^{+}<0.7\right)$. Since the coalescence rate is an increasing function of $\delta U$, it explains the higher rate of coalescence in upward flow.

At microgravity conditions, previous experiments with the same facility (Colin et al. 1991) have shown that the bubble size drastically increases along the pipe because of coalescence: the bubble velocity is almost insensitive to their size and the time of contact during bubble-bubble collisions can be large thus promoting the collision efficiency. As it seemed impossible to prevent the flow development the bubble coalescence was inhibited for the microgravity experiments. This allowed minimizing of the effect of gravity fluctuations on the radial motion of the bubbles (see $\$ 2.3$ ) and obtaining comparable bubble sizes for both laboratory and microgravity experiments. 
This was done by adding $1.6 \times 10^{-3} \mathrm{~mol} \mathrm{~m}^{-3}$ of sodium dodecyl sulphate to the tap water. This concentration is smaller than the minimal concentration of surfactant of $0.05 \mathrm{~mol} \mathrm{~m}^{-3}$ at which drag reduction was observed for a $2 \mathrm{~mm}$ diameter air bubble rising in still water (Duineveld 1994). At microgravity conditions the concentration of surfactant required to immobilize the interface is expected to be still greater than for terrestrial experiments so that the Marangoni effect promoted by the transport of surfactant at the bubble surface was negligible in our case. The mean bubble diameter measured by image processing for the different runs is equal to $1.8 \mathrm{~mm}$ for the run $\mathrm{M} 1,1.2 \mathrm{~mm}$ for run M4 and $2 \mathrm{~mm}$ for run M3 (table 1).

\subsection{Vertical upward flow}

The results of the runs $\mathrm{U} 1, \mathrm{U} 3, \mathrm{U} 4$ obtained at $x / D=70$ are plotted in figure 4 . The radial distributions of the local gas fraction $\alpha$, the Sauter diameter $d$, the mean velocity $U$ and the r.m.s. velocity $u^{\prime}$ of both phases are displayed on these graphs. In these experiments, $R i_{*}$ ranges between 0.6 (U4) and 2.8 (U1). Thus for U1 the effect of buoyancy should be dominant, whereas for $\mathrm{U} 4$ it should be small. With $R i_{*}=1.5$, U3 is an intermediate case. Note that for U4, the gas velocities and the bubble diameters were not determined near the pipe centre because the gas fraction was too small and the time required for the statistical averages to converge was not reached.

For the different runs, the mean diameter ranges mostly between 3 and $4 \mathrm{~mm}$. A slight increase is observed near the pipe wall where the gas fraction is maximum and where bubble coalescence is expected to take place. The gas fraction profiles exhibit a classical peak near the wall. For U3 and U4, the maximum value in the gas fraction profiles is located at a distance from the wall of $\sim 0.5 \mathrm{~d}$ and the thickness of the gas fraction peak is $\sim d$. A bubble layer moves along the wall. These gas fraction profiles display two separate regions: a bubble layer along the wall and a core region $\left(r^{+}<0.7\right)$ with a low or negligible (U4) value of the gas fraction. For the lower liquid velocity (run U1) and higher values of $R i_{*}$, the gas fraction profile is relatively flat with a small peak near the wall: the two regions are not clearly visible.

The present results are compared in figure 4 to the data of Liu \& Bankoff (1993a) obtained in a pipe of diameter $D=38 \mathrm{~mm}$ at a distance $x=36 D$ from the mixer for $j_{L}=0.97 \mathrm{~m} \mathrm{~s}^{-1}, j_{G}=0.027 \mathrm{~m} \mathrm{~s}^{-1}$. These conditions as well as the resulting value of gas fraction $\langle\alpha\rangle=0.02$ are close to those of U4. Although the mean bubble diameter is smaller in the experiments of Liu \& Bankoff (1993a) than in our experiments, the gas fraction profiles are almost the same: the gas fraction is very small in the inner half of the pipe and the location of the maximum is nearly the same although it is slightly closer to the wall because the bubbles are smaller.

The profiles of $U_{L}$ and $U_{G}$ are plotted in figure 4, together with the mean velocity distribution of the liquid in single-phase flow. As already reported by other researchers, the velocity profiles are flatter in two-phase flow than in single-phase flow. On the one hand, the eddy viscosity may be higher in two-phase flow as the turbulence level further suggests. On the other hand the greater velocity of the liquid near the wall may be attributed to the momentum transfer from the fast-moving bubbles to the liquid. Consequently, near the pipe centre, the velocity of the liquid is smaller than in single-phase flow. The mean velocities measured by Liu \& Bankoff (1993a) are slightly greater than those obtained for $\mathrm{U} 4$, except near the wall. The velocity difference between phases, $U_{G}-U_{L}$, is equal to about $0.25 \mathrm{~m} \mathrm{~s}^{-1}$ near the pipe centre. This difference decreases to $0.1 \mathrm{~m} \mathrm{~s}^{-1}$ near the pipe wall where the gas fraction, the mean shear and the turbulence level are greater. Different behaviours of the slip velocity are found in the literature: in the Liu \& Bankoff (1993b) measurements it 


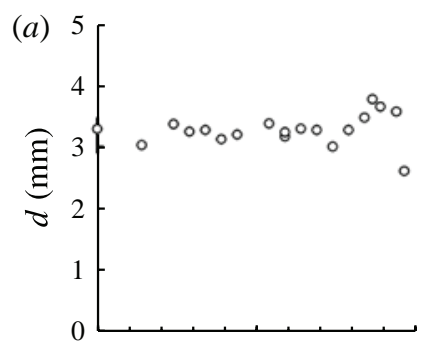

(b)
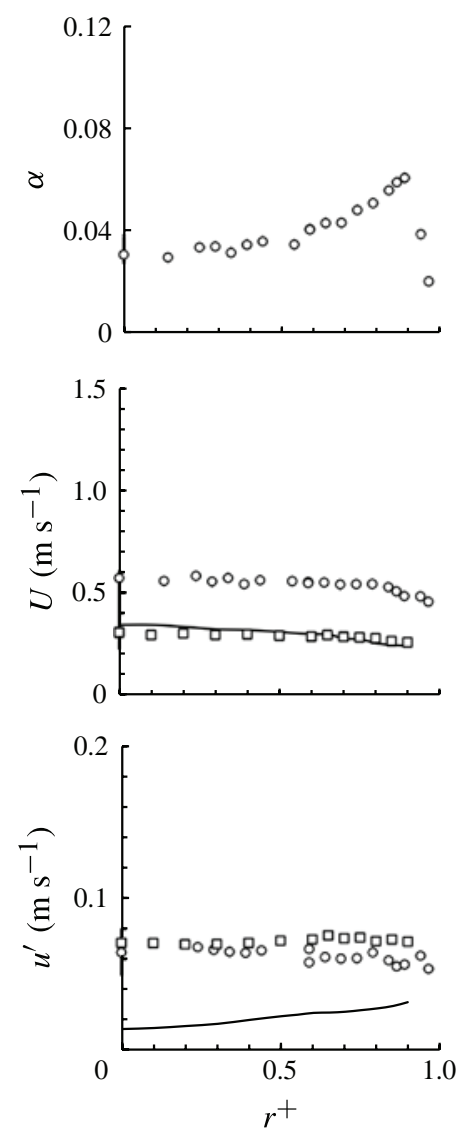
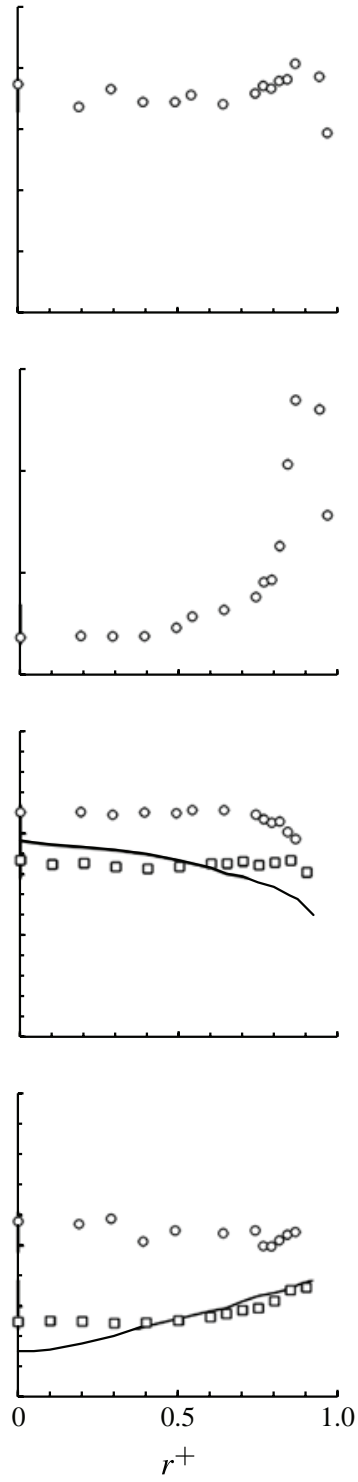

(c)
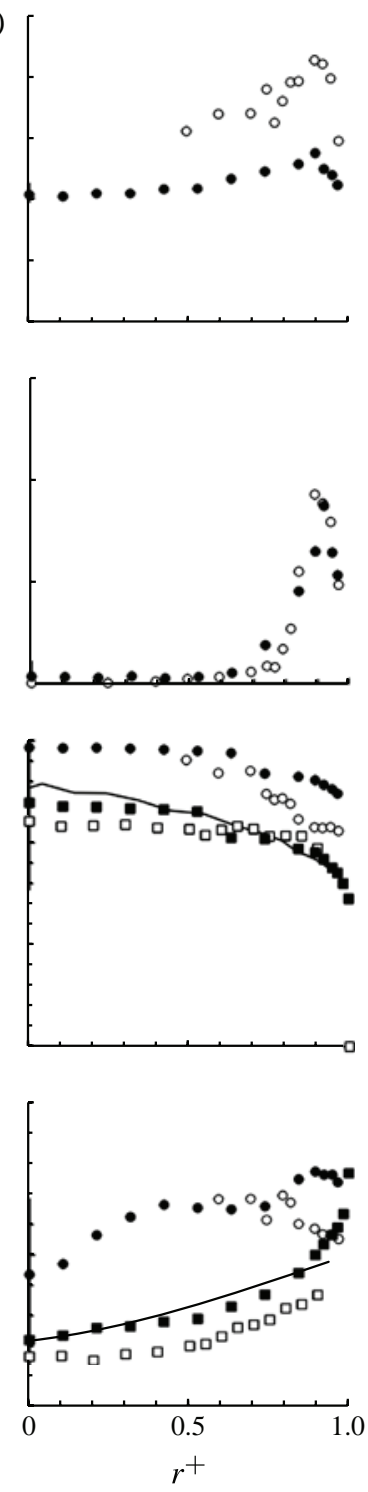

FIGURE 4. Upward flow. From top to bottom: bubble diameter, gas fraction, mean velocity and r.m.s. velocity, for gas (circles), and liquid (squares). Present study: open symbols or solid lines for single-phase flow; Liu \& Bankoff (1993a): solid symbols. (a) U1 : Re = 11 700; (b) $\mathrm{U} 3: \operatorname{Re}=32640 ;(c) \mathrm{U} 4: \operatorname{Re}=40920$.

increases near the wall whereas in the measurements of Grossetête (1995), Serizawa et al. (1975) and Hosokawa \& Tomiyama (2004) it decreases near the wall. This will be discussed in $\S 6$.

The profiles of the r.m.s. velocities of the liquid $u_{L}^{\prime}$ and the gas $u_{G}^{\prime}$ are also plotted in figure 4 together with the values of $u_{L}^{\prime}$ in single-phase flow. In the core region, the profiles are flatter in two-phase flow than in single-phase flow. For the run U1 dominated by buoyancy, the r.m.s. velocity of the liquid is much greater than in 
single-phase flow. It is in fact comparable to the r.m.s. velocity of the gas. In this case the turbulence induced by the bubble motion is greater than the turbulence produced by the wall shear stress as suggested by the high value of $R i_{*}$. For U3 the turbulence level is close to that measured in single-phase flow. For U4, at high liquid flow rate and low gas fraction, the bubble-induced turbulence is weak and the turbulence level is smaller than in single-phase flow. The reduction of turbulence in two-phase flow at high liquid velocity was also shown by other authors (e.g. Serizawa et al. 1975) and will be discussed in $\S 6$. The r.m.s. velocities in the liquid phase found by Liu \& Bankoff $(1993 a)$ are greater than those obtained in the present study for U4. This difference may be explained by the fact that the bubble size is not the same in both experiments. However the data processing of the hot-wire anemometry signal may also be responsible for this difference. Indeed the determination of the r.m.s. velocities of the liquid is very sensitive to the phase discrimination method, which is not the same in the two experiments. Therefore in the present study, in all the runs the same signal processing is used whatever the gravity level.

The r.m.s. velocity of the gas phase $u_{G}^{\prime}$ displays a fairly uniform radial distribution. Its value increases with the gas mean velocity. The ratio $u_{G}^{\prime} / u_{L}^{\prime}$ is equal to about 1 for U1 where the bubble-induced turbulence is high. For the other cases, this ratio is greater: close to 3 for U3 and 2.5 for U4. The values of $u_{G}^{\prime}$ found by Liu \& Bankoff $(1993 b)$ are slightly greater than in our experiments, especially near the pipe wall.

\subsection{Vertical downward flow}

For vertical downward flows, only two runs are complete enough to be presented in this section (D3 and D4 of table 1) but other runs (D2, D2a, D3a and D4a) of Kamp (1996) have been selected and will be included in the discussion in $\S 6$. D3 and D4 correspond to $R i_{*}=1.8$ and 0.7 respectively. As the liquid velocity is smaller than $0.4 \mathrm{~m} \mathrm{~s}^{-1}$, the bubble velocity becomes very small because the bubble slip velocity is opposite and about equal to the liquid velocity. Thus the rate of bubble coalescence is high and some large bubbles tend to have an ascending motion. The results presented in figure 5 were obtained at a distance from inlet of $x / D=70$, as for upward flow. The present results are compared to the measurements of Nakoryakov et al. (1996) revisited by Kashinsky \& Randin (1999) performed in a $42.2 \mathrm{~mm}$ pipe: D3 and D4 have been compared to their results corresponding to flow conditions $d=1.48 \mathrm{~mm}, j_{L}=0.75 \mathrm{~m} \mathrm{~s}^{-1}$ and $j_{G}=0.043 \mathrm{~m} \mathrm{~s}^{-1}$ and $d=1.7 \mathrm{~mm}, j_{L}=1 \mathrm{~m} \mathrm{~s}^{-1}$ and $j_{G}=0.02 \mathrm{~m} \mathrm{~s}^{-1}$ respectively.

The bubble size distribution is almost uniform with the distance from the axis, the Sauter diameter being $\sim 3 \mathrm{~mm}$ (run D4) and $4 \mathrm{~mm}$ (run D3). As the greater value corresponds to the greater gas fraction this suggests the role of coalescence, which increases with the gas fraction.

The gas fraction profiles in figure 5 present a maximum near the pipe centre. The void peaking observed in upward flow is absent (run D4) or attenuated (run D3). In the present case the lift force is directed towards the pipe axis so that the bubbles accumulate in the central region of the pipe. The presence of a second maximum near $r^{+}=0.7$ in D3 has already been observed by other authors for high gas fraction (Wang 1985). As in upward flow, the gas fraction profiles display two regions: a core region with an almost homogeneous value of the gas fraction and a wall region completely free of bubbles. The transition between these two regions is quite sharp. In the experiments of Kashinsky \& Randin (1999) the gas fraction distribution slightly differs from the profile of run D4: the bubbles are less concentrated in the inner region of the pipe. This difference is attributed to the bubble size: in our experiments the 


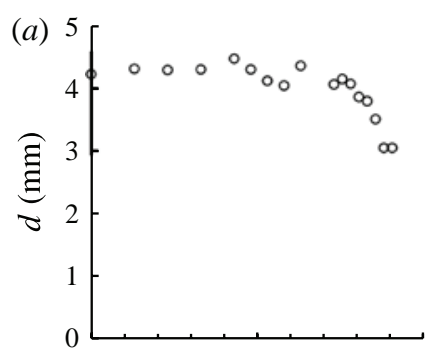

(b)
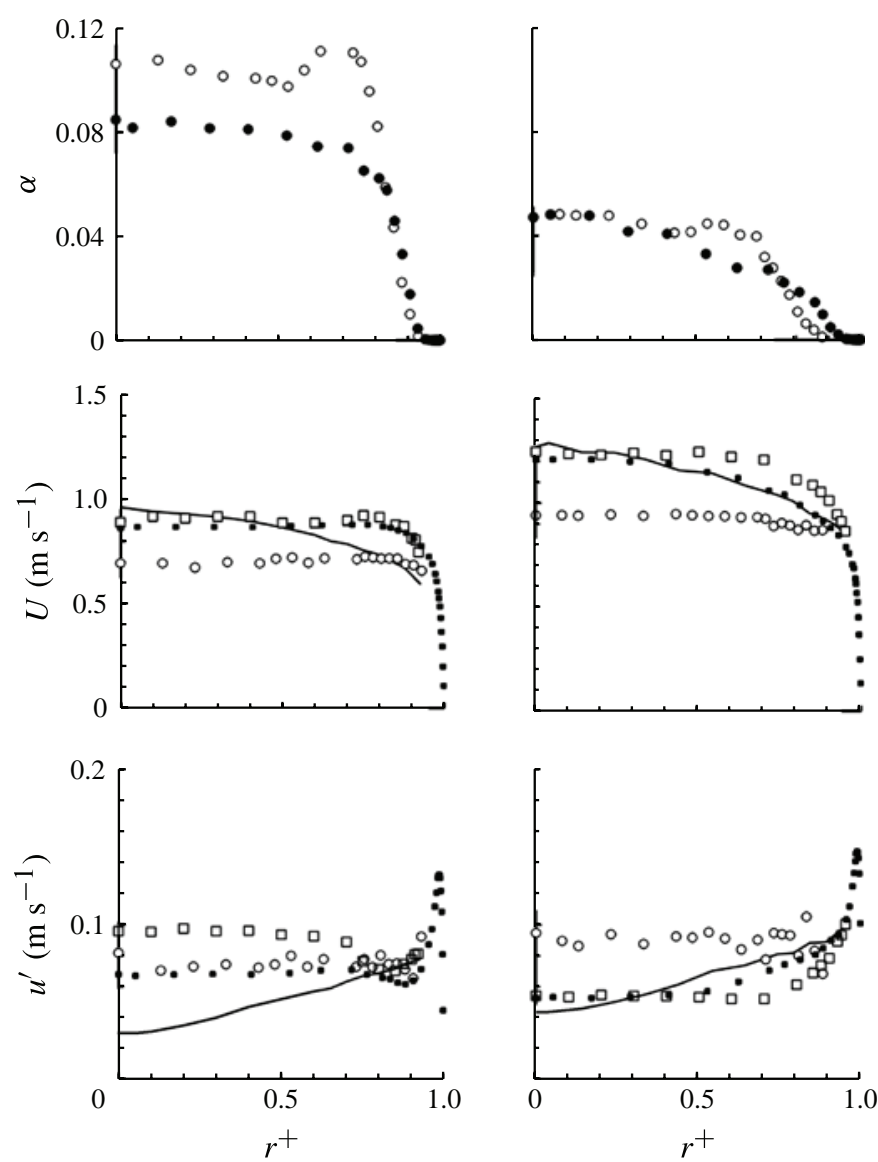

FIgURE 5. Downward flow. From top to bottom: bubble diameter, gas fraction, mean velocity and r.m.s. velocity, for gas (circles), and liquid (squares). Present study: open symbols or solid lines for single-phase flow; Kashinsky \& Randin (1999): solid symbols. (a) $\mathrm{D} 3: R e=32$ 920; (b) D4 : Re $=40960$.

bubble diameter is $\sim 3 \mathrm{~mm}$ against $1.7 \mathrm{~mm}$ in the experiments of Kashinsky \& Randin (1999).

As in upward flow, the mean velocity profiles of the liquid are also flatter in downward flow than in single-phase flow (figure 5). The liquid is slowed down by the bubbles in the central region of the pipe. Like the gas fraction, the results of D3 display a maximum velocity for $r^{+} \approx 0.7$. In contrast to the results of D4 
the measurements of Kashinsky \& Randin (1999) show that the profiles measured in single- and two-phase flow are very close. Again, this difference with our measurements seems to be due to their smaller bubble diameters. Similar velocity profiles observed for D3 are also observed in the results of Kashinsky \& Randin (1999) even though the gas velocity is not exactly as in D3. They also observed the flattening of the mean velocity profile for higher gas fractions.

The bubble slip velocity varies between $-0.3 \mathrm{~m} \mathrm{~s}^{-1}$ near the pipe centre and $-0.15 \mathrm{~m} \mathrm{~s}^{-1}$ closer to the wall. As in vertical upward flow, a decrease of the bubble slip velocity is measured near the wall.

For the two runs, different r.m.s. velocity profiles are observed in the liquid (figure 5). In D3, the distribution of $u_{L}^{\prime}$ is almost uniform: except near the wall where the bubbles are absent the liquid turbulence is higher than in single-phase flow suggesting the role of the bubble slip. The flow is dominated by the bubble-induced turbulence as the value of $R i_{*}$ suggests. This effect is also observed in the experiments of Kashinsky \& Randin (1999) but less pronounced because of lower gas fraction. In D4, the values of $u_{L}^{\prime}$ are comparable to those observed in single-phase flow with some differences: for $0.4<r^{+}<0.9, u_{L}^{\prime}$ is much smaller than in single-phase flow. These trends were also observed by Kashinsky \& Randin (1999) with smaller bubble diameter. In the core region the profiles of $u_{L}^{\prime}$ are flatter for D3 and D4 than in single-phase flow.

Like the liquid phase, D3 and D4 display a different behaviour of the r.m.s. velocities of the gas phase: as usually observed $u_{G}^{\prime}$ is much greater than $u_{L}^{\prime}$ in run D4 while for run D3 the turbulence of the gas phase is comparable to that of the liquid phase. These features were already pointed out for upward flow. We must note in passing that in run D3 the turbulence of the gas phase is in fact $20 \%$ lower than the turbulence in the liquid phase. This result is rather unexpected.

\subsection{Microgravity flow}

For flow at microgravity conditions the effect of buoyancy is suppressed. Only some disturbing effects of the $g$-jitter remain that are difficult to suppress in parabolic flights. The most specific difference with upward or downward flow is the fact that the bubbleinduced turbulence should disappear. Because $R i_{*}$ is nearly zero the flow is dominated by the shear-induced turbulence. Three runs (M1, M3 and M4 of table 1) are presented in this paper. For these experiments the bubble size could not be measured because the available time was too short for the statistics to converge. Nevertheless a typical bubble size was obtained from image processing. The radial distributions of gas fraction, mean and r.m.s. velocity of both phases are plotted in figure 6 .

The gas fraction profiles present a shape quite similar to that of downward flow. However, the profiles are flatter and more homogeneously distributed in microgravity: there is no outer-half region without bubbles like in downward flow. For M3 and M4, the shapes of the profiles in the two experiments are very close. For M1 a difference is observed: in this run, the gas fraction is unusually high near the pipe centre and low elsewhere. We verified the measurement accuracy (see $\S 2.1$ ): it appears that though $\langle\alpha\rangle_{m}$ and $\langle\alpha\rangle$ are in reasonable agreement for M3 and M4, $\langle\alpha\rangle_{m}$ is twice as large as $\langle\alpha\rangle$ for M1. This discrepancy between the cross-sectional average and the volumetric measurement is attributed to a perturbation of the radial motion of the bubbles due to the residual acceleration ( $g$-jitter) during the short period of microgravity, as it was explained in $\S 2.3$.

In two-phase flow, the velocity profiles of the liquid are similar to single-phase flow although they are slightly flatter. The most important feature is that the mean 

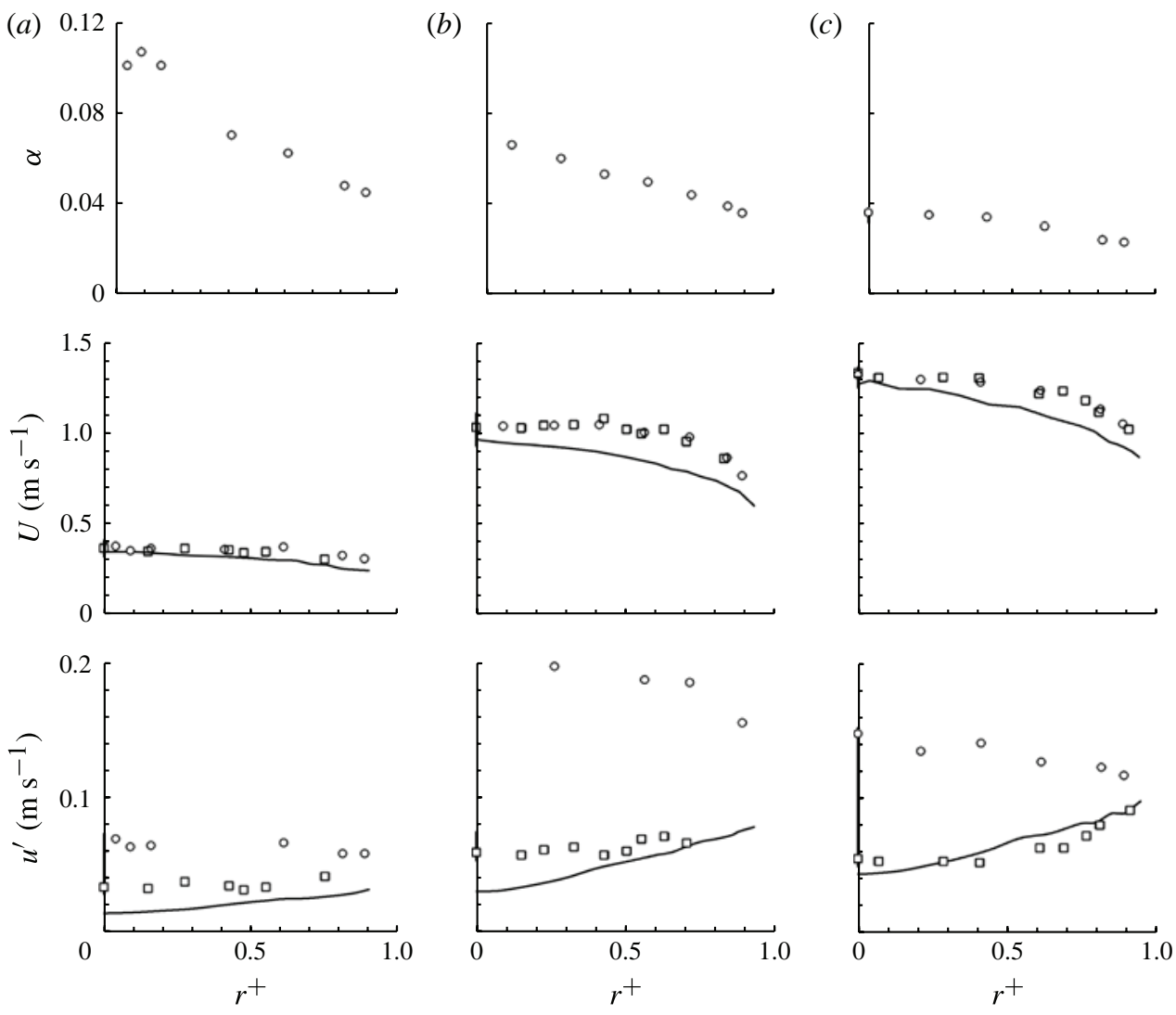

FIGURE 6. Microgravity flow. From top to bottom: gas fraction, mean velocity and r.m.s. velocity, for gas (circles) and liquid (squares). Present study: open symbols or solid lines for single-phase flow. (a) M1:Re=12000; (b) M3 : Re=33 040; (c) M4:Re=41 120.

gas velocity is equal to that of the liquid, as expected in the absence of buoyancy. Lahey \& Bonetto (1994) obtained similar results with neutrally buoyant particles. The bubble slip velocity cannot be determined because it is smaller than the measurement uncertainty. As explained in $\S 2.1 j_{L}$ and $j_{G}$ are compared to $\left\langle(1-\alpha) U_{L}\right\rangle$ and $\left\langle\alpha U_{G}\right\rangle$ respectively. Whereas they should be equal, $\left\langle\alpha U_{G}\right\rangle$ is $20 \%$ to $30 \%$ smaller than $j_{G}$ for M1 and M3, pointing out the perturbation induced by the residual gravity.

The r.m.s. velocity of the liquid is close to or slightly greater than that measured in single-phase flow. The comparison of runs $\mathrm{U} 1$ and M1 corresponding to similar flow rates clearly shows the role of buoyancy in the turbulence of the liquid phase. While the turbulence is controlled by the slip velocity in upward flow, it becomes shear dependent when the gravity is suppressed.

Finally the r.m.s. velocity of the gas phase in figure 6 is twice to three times greater than the turbulence in the liquid phase. This is also a specific behaviour of microgravity flow.

\section{Discussion}

In single-phase turbulent pipe flow, both inertia and viscous forces control the fluid motion so that the flow is completely characterized by the ratio of these two 
forces: the Reynolds number. In two-phase flow the scaling is far more complex and does not reduce to one dimensionless number, not only because there are two phases with different physical properties and volumetric flux, but also because another force comes into play: the buoyancy. Since the present experiments extend over a wide range of $R i_{*}$, it is possible to demonstrate its contribution. But its parameterization would require results from many more flow conditions than those of the present study. Unfortunately the published data are often incomplete, most of the time because the wall friction has not been determined. Thus no additional data are available, except those of Nakoryakov et al. (1996) revisited by Kashinsky \& Randin (1999). The method that we adopted here is to compare our data to existing analytical models to highlight the length or velocity scales that control the physics.

\subsection{Preliminary remark}

As will become obvious hereafter, the friction velocity $u_{*}$ has to be known, not only because it is used in $R i_{*}$ to compare buoyancy and friction, but also because it is essential for the understanding of various aspects of two-phase flow in pipes. Thus it must be determined with an acceptable accuracy. In single-phase flow, various methods exist for determining $u_{*}$ from $\tau_{w}$. The wall shear stress can be measured by various techniques: flush-mounted hot-film probe, Preston tube, polarography, etc. It can be also determined by indirect methods: log-law fitting, extrapolation at the wall of either the turbulence shear stress or of the turbulence kinetic energy (see a comparison of these methods in Fabre, Masbernat \& Suzanne (1983)).

In vertical fully developed bubbly flow, the wall shear stress can be determined from (2.1) if both pressure gradient and gas fraction have been measured. However, in this equation $\mathrm{d} P / \mathrm{d} x$ and $\rho_{L} \gamma(1-\langle\alpha\rangle)$ are close to each other and their difference $4 \tau_{w} / D$ is so small that it is difficult to measure with great accuracy. Nevertheless this method has been selected in the present study and special care has been taken to determine $\mathrm{d} P / \mathrm{d} x$ and $\langle\alpha\rangle$ as accurately as possible.

Additional results would have been welcome but in most previous studies, either the pressure gradient was missing or some doubt exists regarding its accuracy or that of the gas fraction. In principle another indirect method developed in single-phase flow could be used. But log-law fitting is impossible since we know little about its validity in two-phase flow. All that remains is the extrapolation of the turbulence shear stress at the wall: using this method would allow consideration of the rich set of data of Liu (1989). We tried to do this but unlike fully developed single-phase flow where the shear stress distribution varies linearly with the radial coordinate, we can say nothing regarding two-phase flow: its gradient increases with $r$ so much that the near-wall data are too sparse for the wall shear stress to be determined accurately.

\subsection{Liquid mean velocity}

Fully developed turbulent single-phase flow in pipes can be viewed as a three-layer flow. These layers are, from the wall to the axis: the viscous, the inertial and the wake layers. When the Reynolds number increases the inertial layer grows to the detriment of both the viscous and the wake layers. In the inertial layer the velocity follows the logarithmic law expressed for a smooth regime by:

$$
\frac{U_{L}}{u_{*}}=\frac{1}{\kappa} \log \left(\frac{y u_{*}}{v}\right)+B,
$$

where $y$ is the distance from the wall, $\kappa=0.41$ the von Kármán constant and $B=5.5$ an additive constant. In the wake layer the velocity is slightly greater than that 

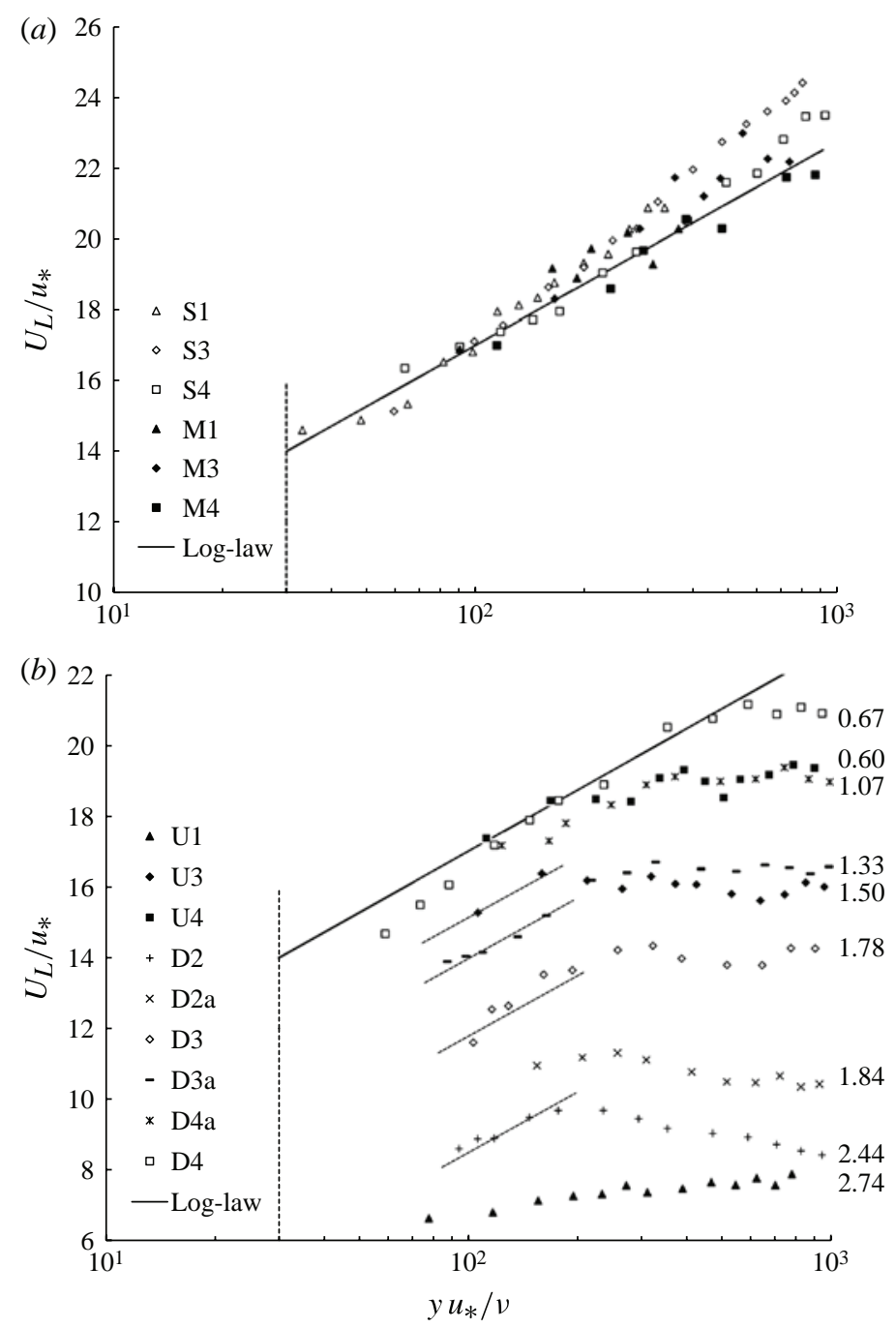

FIGURE 7. Velocity distributions for various runs compared to the log-law (6.1) (-): (a) $R i_{*}=0 ;(b) R i_{*}>0$. The figures at the far right of $(b)$ are the values for $R i_{*}$.

predicted by the log-law: the additional velocity is modelled by a wake function, hence the name of the layer.

From an experimental study of a bubbly boundary layer in upward flow Marié, Moursali \& Tran-Cong (1997) showed that the liquid velocity follows the log-law in the inertial layer. However the von Kármán constant has to be modified for (6.1) to fit the experiments. What about its validity for bubbly flow in a pipe? From the experimental data in upward flow (Nakoryakov et al. 1981), Marié et al. (1997) found that the log-law persists with a decrease of its slope for a gas fraction smaller than $10 \%$ in the core region. In contrast, from the data in downward flow (Nakoryakov et al. 1996) they showed that the log-law persists without any modification of the von Kármán constant.

The present results are plotted in figure 7 in wall coordinates, $y^{+}=y u_{*} / v$ and $U^{+}=U_{L} / u_{*}$. We distinguished the $R i_{*}=0$ flow conditions (figure $7 a$ ) from the $R i_{*}>0$ 

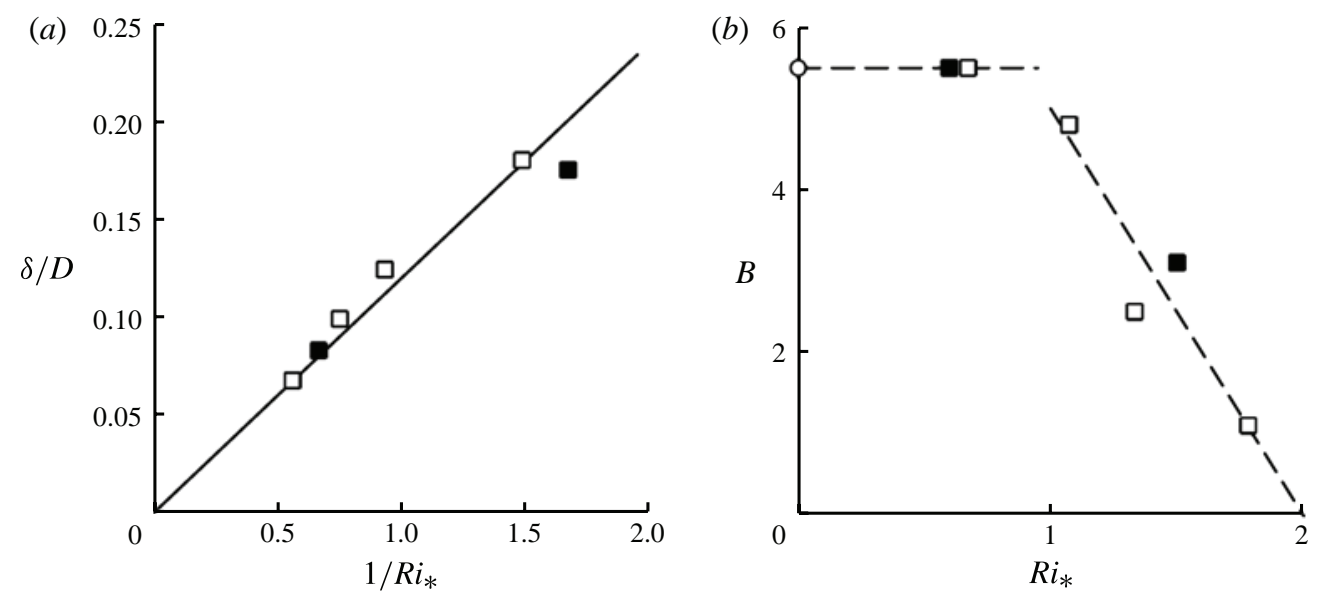

Figure 8. (a) Thickness of the wall layer; (b) constant of the log-law. $\square$, Upward; $\square$, downward; and $\bigcirc$, microgravity flows.

ones (figure $7 b$ ): thus figure 7(a) includes single-phase and microgravity flows whereas figure $7(b)$ includes upward and downward flows.

In the absence of buoyancy (figure $7 a$ ), the liquid velocity displays the same behaviour in single-phase and in two-phase flows: there exists a wall layer where the log-law (6.1) is valid, and a wake region. Thus in the absence of gravity the structure of the liquid flow is not sensitive to the presence of bubbles.

For gravity-dependent flows (figure $7 b$ ) the velocity distribution presents an important difference. If an inertial layer were to exist as for $\gamma=0$, the wake layer would be replaced by a core flow where the velocity is nearly constant. Thus we will talk about a core region rather than a wake region: it will be shown further that the homogeneity of the flow in that region concerns most of the physical quantities. In the inertial layer located in the wall region, the validity of the log-law depends on the value of $R i_{*}$. When $R i_{*}<1$ the log-law is still valid even though its $y^{+}$-range of validity decreases when $R i_{*}$ increases. When $R i_{*}>1$ the available data are insufficient to conclude safely on the validity of the log-law. Nevertheless we believe that it remains valid with the same slope in the semi-log representation of figure 7 . We observe also that when $R i_{*}$ increases above unity, the $y^{+}$-range of constant velocity increases to the detriment of the wall layer. The ratio of the core diameter to the wall layer thickness quantifies the relative importance of buoyancy and friction. The existence of these two regions, a wall layer and a core, is an important feature of gravity-dominated two-phase flows that will be confirmed below. We made a crude estimate of the wall layer thickness $\delta$, plotted in figure 8(a). According to the present results $\delta / D$ is proportional to $R i_{*}^{-1}$, yielding

$$
\delta=0.48 \frac{u_{*}^{2}}{\langle\alpha\rangle|\gamma|} .
$$

This correlation, which works equally well in upward and in downward flow, suggests that the thickness of the inertial layer that persists in two-phase flow is independent of the pipe diameter and results from a balance between friction and buoyancy. This conclusion is in contradiction with previous studies, and in particular that of Liu \& Bankoff (1993a) that state that $\delta$ is controlled by the bubble diameter $d$. But it 
would be surprising if the layer thickness were controlled by the bubble size since in downward flow there are no bubbles in that layer.

Let us come back to the log-law and its parameters $\kappa$ and $B$. Since the slope of the velocity distribution does not change, the von Kármán constant $\kappa$ keeps the value 2.5 that it takes in single-phase flow. While $\kappa$ remains unchanged in two-phase pipe flow, the conclusion is different for $B$. As figure $8(b)$ shows, the additive constant decreases for $R i_{*}>1$. Its behaviour is well-represented by

$$
B=5.5-5\left(R i_{*}-1\right) \mathrm{H}\left(R i_{*}-1\right),
$$

where $\mathrm{H}$ is the Heaviside function. It is remarkable that the behaviour of $B$ changes at $R i_{*}=1$ : it could have justified the choice of the buoyancy number $R i_{*}$ but others dimensionless numbers involving a velocity scale different from $(|\gamma| D)^{1 / 2}$ (e.g. $(|\gamma| d)^{1 / 2}$ or $\left.U_{L G}\right)$ would have been possible. Although Marié et al. (1997) observed a modification of $\kappa$ in boundary layer flow, Hosokawa, Suzuki \& Tomiyama (2010) confirm for duct flow the trends that we observe for $\kappa$ and $B$.

Why is the additive constant modified in two-phase pipe flow? The answer is not clear. In single-phase flow a general expression for $B$ that includes the effect of the wall roughness $k_{s}$ is (Fabre et al. 1983)

$$
B=5.5-\frac{1}{\kappa} \log \left(1+0.3 \frac{k_{s} u_{*}}{v}\right) .
$$

For a hydraulically smooth wall $B$ equals 5.5 whereas it decreases when the roughness Reynolds number $k_{s} u_{*} / v$ increases. In two-phase flow, the decrease of $B$ has sometimes been interpreted as due to the roughness induced by the bubbles near the wall. But this explanation does not hold because (6.3) also fits the results in downward flow where the wall layer is almost free of bubbles. Since the critical value of $R i_{*}$ above which $B$ starts to decrease is close to unity, one can infer that, when the buoyancy effect dominates, the core layer generates fluctuations imposed on the wall layer like waves imposed by the gas on the liquid film in annular flow. This is of course an open question that two-phase flow codes may help to close.

\subsection{Turbulent shear stress}

Because we used a single probe in our experiments the shear stress was not measured. However it can be deduced from the $x$-component of the mixture momentum assuming that the flow is fully developed and that the mean and turbulent contributions of the gas inertia remain negligible. With these assumptions, the $x$-component of the momentum balance can be written

$$
-\frac{\mathrm{d} P}{\mathrm{~d} x}+\frac{1}{r}\left[r(1-\alpha) \tau_{L}\right]-\rho_{L}(1-\alpha) \gamma=0,
$$

where the shear stress $\tau_{L}$ contains viscous and turbulent contributions:

$$
\tau_{L}=\rho_{L} \nu \frac{\mathrm{d} U_{L}}{\mathrm{~d} r}-\rho_{L} \overline{u_{L} v_{L}} .
$$

Integrating (6.5) with respect to $r$ and introducing $\tau_{w}$ and $R i_{*}$ leads to

$$
(1-\langle\alpha\rangle) \frac{\tau_{L}}{\tau_{w}}=\frac{r}{R}\left[1 \mp R i_{*}\left(1-\frac{\langle\alpha\rangle_{0}^{r}}{\langle\alpha\rangle}\right)\right],
$$

where $\langle\alpha\rangle_{0}^{r}$ is the average gas fraction within an inner cylinder of radius $r$. Note that the shear stress $\tau_{L} / \tau_{w}$ is positive even if the two quantities $\tau_{w}$ and $\tau_{L}$ are negative. In 

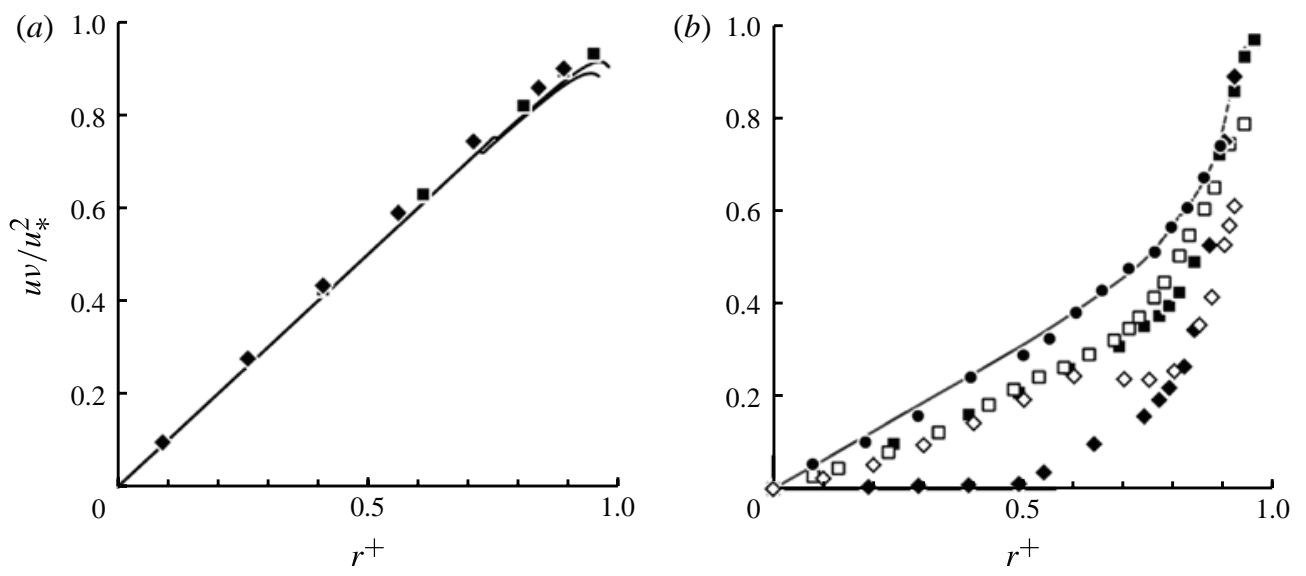

FIGURE 9. Dimensionless turbulent shear stress calculated from equation (6.7). (a) $R i_{*}=0$ : - , S3 and S4; $\diamond, \mathrm{M} 3 ; \mathbf{\square}, \mathrm{M} 4 .(b) R i_{*}>0: \diamond, \mathrm{D} 3 ; \square, \mathrm{D} 4 ; \diamond, \mathrm{U} 3 ; \mathbf{\square}, \mathrm{U} 4 ;-$, Liu \& Bankoff (1993a) experiment; and $\boldsymbol{O}$, calculation from (6.7).

addition, since $\gamma>0$ (respectively, $<0$ ) for upward (respectively, downward) flow, the $\mp$ sign in the bracketed term of (6.7) is negative (respectively, positive) to account for the definition of $R i_{*}$.

In single-phase flow $\tau_{L} / \tau_{w}$ is linear with respect to $r$. But in two-phase flow there exist only two specific cases for which $(1-\alpha) \tau_{L} / \tau_{w}$, and not $\tau_{L} / \tau_{w}$, has the same property: (i) for the improbable case of a homogeneous gas fraction distribution and (ii) at microgravity conditions because $R i_{*}=0$. These cases apart, $(1-\alpha) \tau_{L} / \tau_{w}$ as well as $\tau_{L} / \tau_{w}$ are not linear with respect to $r$.

If the distribution of $\alpha$ is known $\tau_{L}$ may be determined from (6.7). Except in the near-wall region the viscous stress in (6.6) is negligible and the shear stress is nearly equal to the turbulent shear stress. An equation equivalent to (6.7) was used by Wang (1985) to estimate the gas fraction distribution from the turbulent shear stress measurements. He found a good agreement with the measured values in a downward bubbly flow. In the present study, we used (6.7) to calculate the shear stress from the gas fraction. The method was validated from the results of Liu \& Bankoff (1993a) by comparing their predicted and measured values (figure 9). However it was necessary to calculate $\tau_{L}$ from smooth distributions of $\alpha$ and $U_{L}$. The distribution of the turbulent contribution $\overline{u_{L} v_{L}} / u_{*}^{2}$ is displayed in figure 9. As we did for the velocity distribution, we separated the $R i_{*}=0$ conditions (figure $9 a$ ) from the others (figure $9 b$ ) to demonstrate the gravity effects.

If $R i_{*}=0,(1-\alpha) \tau_{L} / \tau_{w}$ must be linear with respect to $r$. So must be $(1-\alpha) \overline{u_{L} v_{L}} / u_{*}^{2}$, apart from the wall region. This is clearly the case for the two single-phase flow conditions: the solid lines are indeed indistinguishable (figure 9a), except in the viscous sub-layer where the turbulent shear stress becomes negligible with respect to the viscous counterpart. For two-phase flow at microgravity conditions, the linearity of $(1-\alpha) \tau_{L} / \tau_{w}$ leads to that of $\overline{u_{L} v_{L}} / u_{*}^{2}$ because $\alpha$ is small and almost homogeneous.

For gravity flows (figure $9 b$ ), $\overline{u_{L} v_{L}} / u_{*}^{2}$ is everywhere smaller than in single-phase flow. Moreover the qualitative behaviour of the shear stress distribution is the same in upward and downward flow. This is due to the fact that the square-bracketed term of (6.7) is always smaller than unity. For upward flow the sign before the parenthesis is negative and the gas fraction in a cylinder of radius $r$ is always smaller than that of 
the pipe because the bubble density is greater near the wall. Conversely, for downward flow the sign is positive, as the gas fraction in a cylinder of radius $r$ is greater than that of the pipe because the bubble density is greater at the pipe axis. This shear stress reduction by the presence of bubbles may be understood as follows. In upward flow the inner cylinder is denser than the surrounding annulus: thus it exerts a buoyant force on the annulus reducing by an equal quantity the friction at the inner surface of the annulus. A similar explanation applies to downward flow, considering that friction force and buoyancy are in opposite directions: the greater $R i_{*}$ the smaller $\overline{u_{L} v_{L}} / u_{*}^{2}$. It is important to note that even if $\overline{u_{L} v_{L}} / u_{*}^{2}$ is smaller in bubbly flow than in single-phase flow it is not always the case for $\overline{u_{L} v_{L}}$. Indeed the value of $u_{*}$ may be larger in bubbly flow, especially when $R i_{*}>1$.

The shear stress distribution of gravity flows $\left(R i_{*} \neq 0\right)$ also displays the two-layer structure demonstrated in the previous subsection for gravity flows: a central core where the turbulent shear stress increases slightly with $r$ and a wall region with a sharp gradient. The dimensionless radius of the homogeneous core is equal to about $0.7-0.8$ and seems to have little sensitivity to $R i_{*}$.

\subsection{Turbulence of the liquid phase}

The present results will be discussed and compared to the existing turbulence models, which we recall here. In two-phase flow, turbulence originates from two different mechanisms: (i) the work of friction responsible for the shear-induced turbulence and (ii) the bubble slip that generates the bubble-induced turbulence. The first mechanism exists in single-phase flow whereas the second is specific to two-phase flow. Lance \& Lopez de Bertodano (1994) assumed that for low gas fraction the two mechanisms are weakly coupled. As a consequence the turbulence kinetic energy can be expressed as the sum of the shear- and the bubble-induced turbulence, $k_{L}=k_{L S}+k_{L B}$. For fully developed axisymmetric flow and in the limit of zero coupling, the transport equations of $k_{L S}$ and $k_{L B}$ are

$$
\begin{aligned}
& -(1-\alpha) \overline{u_{L} v_{L}} \frac{\mathrm{d} U_{L}}{\mathrm{~d} r}-(1-\alpha) \varepsilon+\frac{1}{r} \frac{\mathrm{d}}{\mathrm{d} r}\left[(1-\alpha) \frac{v_{t}}{\sigma_{k}} r \frac{\mathrm{d} k_{L S}}{\mathrm{~d} r}\right]=0, \\
& \frac{1}{r} \frac{\mathrm{d}}{\mathrm{d} r}\left[(1-\alpha) \frac{v_{t}}{\sigma_{k}} r \frac{\mathrm{d} k_{L B}}{\mathrm{~d} r}\right]+\frac{k_{L B \infty}-k_{L B}}{t_{b}}=0 .
\end{aligned}
$$

In (6.8) the first term is the production of turbulence kinetic energy, the second is the rate of viscous dissipation and the third is the turbulence energy diffusion written in terms of the eddy viscosity $v_{t}$. In (6.9) the first term is the energy diffusion and in the second $k_{L B \infty}$ is the turbulence kinetic energy added to the liquid by bubbles rising at the velocity $U_{\infty}$ in still liquid and $t_{b}$ is the bubble response time defined as

$$
t_{b}=\frac{U_{\infty}}{2 \gamma} .
$$

Equations (6.8) and (6.9) possesses two explicit solutions. That of (6.8) is well known in single-phase flow. Indeed wherever the diffusion term is negligible, production and dissipation balance: this is the case in the inertial layer. Extrapolating the eddy viscosity model $v_{t}=C_{\mu} k_{L S}^{2} / \varepsilon$ from single-phase flow yields that the turbulence kinetic energy and the shear stress are simply proportional:

$$
k_{L S}=\frac{\overline{u_{L} v_{L}}}{\sqrt{C_{\mu}}} .
$$


Equation (6.9) also has a simple solution. If $t_{b}$, the bubble response time, is much smaller than the eddy diffusivity time scale, (6.9) reduces to $k_{L B \infty}=k_{L B}$ : this should be satisfied in the core if $u_{*} U_{L G} /(\gamma D) \ll 1$. The study of Lance \& Bataille (1991) carried out at high bubble Reynolds number, $R e_{B}=d U_{L G} / v$, in uniform bubbly flows shows that $k_{L B \infty}$ can be expressed as

$$
k_{L B}=k_{L B \infty}=f(\alpha) U_{L G}^{2} .
$$

This has been confirmed by Garnier, Lance \& Marié (2001), Larue de Tournemine (2001) and more recently by Riboux, Risso \& Legendre (2010). The measurements of $u_{L}^{2} / U_{L G}^{2}$, which are expected to behave as $k_{L B} / U_{L G}^{2}$, demonstrate that (Garnier et al. 2001)

$$
\left(u_{L} / U_{L G}\right)^{2} \approx \alpha .
$$

These two solutions tell us which mechanism is dominant. The ratio of the bubbleinduced to shear-induced turbulence is expressed at small $\alpha$ by

$$
\frac{k_{L B}}{k_{L S}} \propto \frac{\alpha U_{L G}^{2}}{\overline{u_{L} v_{L}}} .
$$

In two-phase flow, bubble-induced turbulence should be negligible:

(i) at microgravity conditions since $U_{L G} \approx 0$;

(ii) when $R i_{*}$ is small, because $U_{L G}^{2}$ is a function of $\gamma d$ and it can be argued that $k_{L B} / k_{L S}=(d / D) O\left(R i_{*}\right)$;

(iii) when $d / D$ is small.

But even when $R i_{*}$ and/or $d / D$ are small, some flow regions must exist where the bubble-induced turbulence is significant: this is the case where the shear stress is small enough $\left(\overline{u_{L} v_{L}} \ll \alpha U_{L G}^{2}\right)$, i.e. in the core region. This may be confirmed by plotting the production of the shear-induced turbulence, i.e. the first term of (6.8). In dimensionless form it is given by

$$
\Pi^{+}=-(1-\alpha) \overline{u_{L} v_{L}} \frac{\mathrm{d} U_{L}}{\mathrm{~d} r} \frac{D}{2 u_{*}^{3}} .
$$

In single-phase flow (figure 10a) two regions that correspond to the layers already mentioned in $\S 6.2$ can be distinguished: a wall region corresponding to the inertial layer and a central region corresponding to the wake layer. In microgravity a similar trend is observed since the distributions of $(1-\alpha) \overline{u_{L} v_{L}}$ and $U_{L}$ are similar to what they are in single-phase flow. In gravity flow (figure 10b) two layers also exist. But we have seen in $\S 6.2$ that, if an inertial layer exists, the wake layer is replaced by a core layer characterized by a homogeneous velocity distribution. In that layer $\Pi^{+}$vanishes completely (figure 10b): the rising bubbles inhibit the production of shear-induced turbulence by reducing the turbulence shear stress.

What are the consequences for the turbulence kinetic energy? Because we used a single wire probe, we could not determine $k_{L}$. Thus the discussion will be focused on its signature, the r.m.s. $x$-velocity $u_{L}^{\prime}$ that is plotted in figure 11 . The predicted shear-induced turbulence contribution is also plotted. It was estimated as follows: we calculated $k_{L S}$ from (6.11) and $\overline{u_{L} v_{L}}$ given in $\S 6.3$, and then the r.m.s. contribution of the shear-induced turbulence $u_{L}^{\prime} \approx\left(2 k_{L S} / 3\right)^{1 / 2}$ by assuming that turbulence is isotropic. As in the previous subsections, the results are presented by distinguishing the cases corresponding to $R i_{*}=0$ (figure 11a) and the cases corresponding to $R i_{*}>0$ 

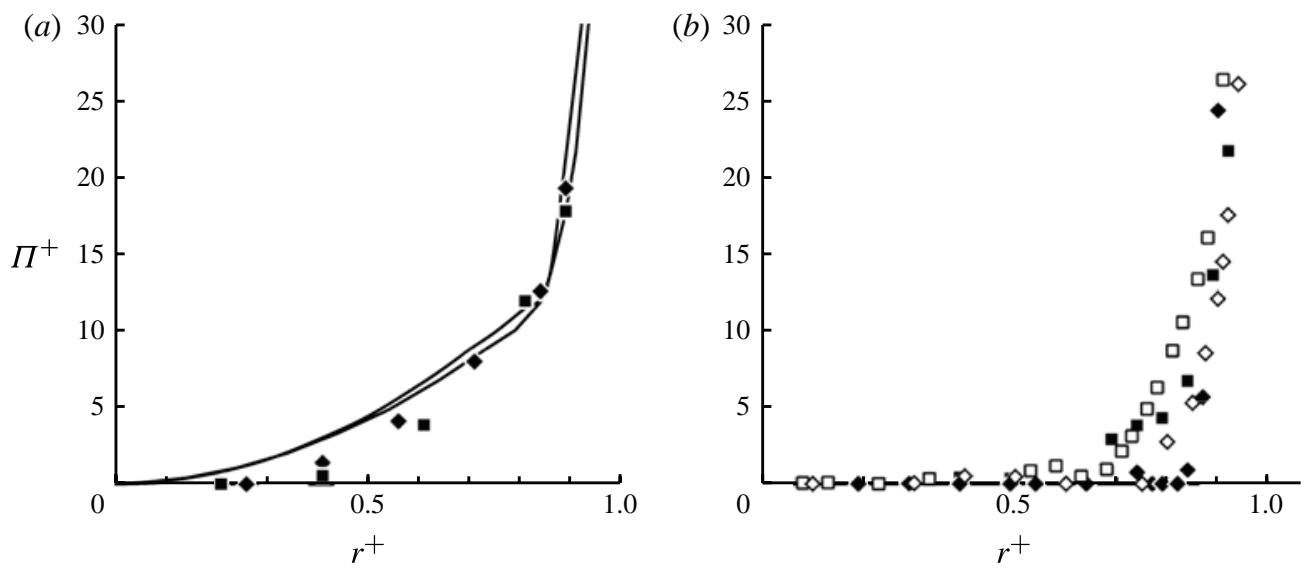

FIGURE 10. Production of shear-induced turbulence kinetic energy. (a) $R i_{*}=0:-, \mathrm{S} 3$ and $\mathrm{S} 4 ; \diamond, \mathrm{M} 3 ; \mathbf{\square}, \mathrm{M} 4$. (b) $R i_{*}>0: \diamond, \mathrm{D} 3 ; \square, \mathrm{D} 4 ; \diamond, \mathrm{U} 3 ; \square, \mathrm{U} 4$.
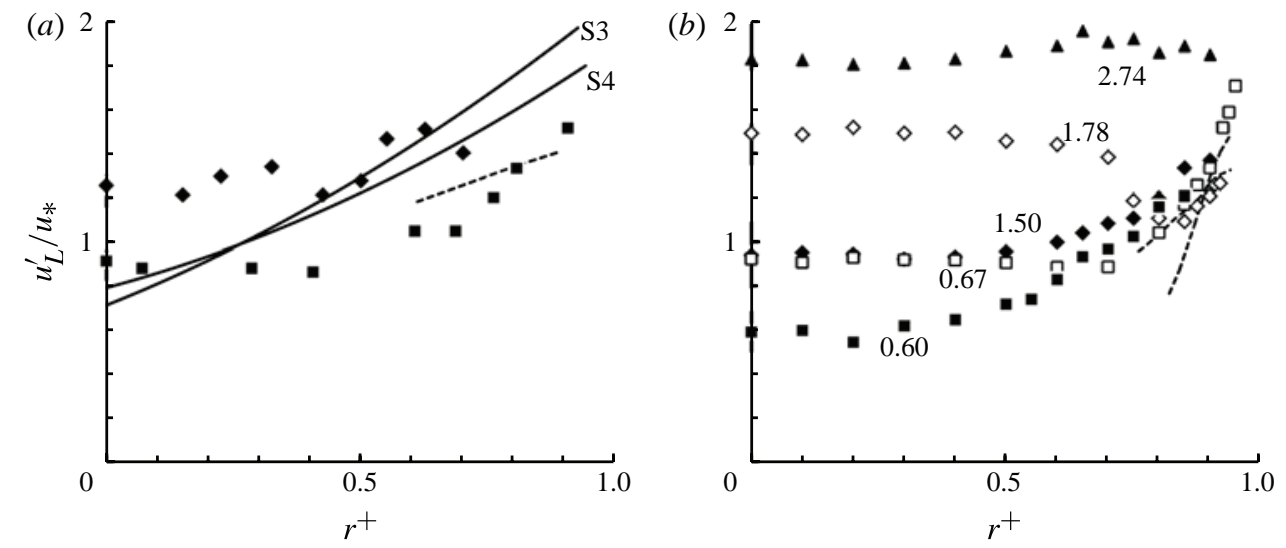

FIgURE 11. Dimensionless r.m.s. velocities of the liquid. (a) $R i_{*}=0$ : (-), S3 and S4; ( ),

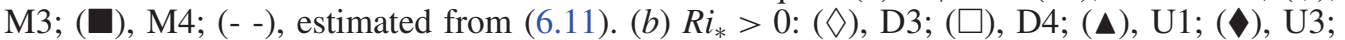
$(\square)$ U4; (- -), estimated from (6.11). The figures adjacent to the plots in $(b)$ are the values for $R i_{*}$. The dashed lines in $(b)$ were claculated for $R e=30000$ to compare to U3 (open diamonds) and $R e=40000$ to compare to U4 (open squares).

(figure 11b). The single-phase flow distributions, considered as a reference, show little sensitivity to the Reynolds number (figure 11a). As expected this is still nearly the case at microgravity conditions because of the absence of bubble-induced turbulence: in that case the liquid phase behaves almost as if it were alone in the pipe. A different behaviour is observed for $R i_{*}>0$ (figure $11 b$ ). To throw light on these results we added the value of $R i_{*}$. If we accept that $u_{L}^{\prime}$ is a good signature of the turbulence intensity, we can draw the following conclusions.

(i) It is not as easy to identify the two layers with $u_{L}^{\prime}$ as it is with $\Pi^{+}$. This is because the shear-induced turbulence goes into the core by turbulent diffusion. Nevertheless figure 11(b) with the exception of run U1 shows the existence of a wall layer where $u_{L}^{\prime} / u_{*}$ is almost independent of $R i_{*}$. 
(ii) For upward and downward flows the turbulence in the core increases with $R i_{*}$ : the greater $R i_{*}$ the larger the effect of buoyancy on bubble-induced turbulence.

(iii) For given $R i_{*}$ the turbulence level in the core is smaller in upward flow than in downward flow. Why is it so? On the one hand there are fewer bubbles in upward flow than in downward flow and there is less bubble-induced turbulence. On the other hand the shear-induced turbulence is created near the wall and part of it goes into the core through turbulent diffusion: in upward flow a smaller amount is produced near the wall because there is less liquid and the amount of turbulence transferred through turbulent diffusion has to fill a region that contains more liquid.

(iv) The turbulence level in the core can be lower in two-phase flow than in single-phase flow (e.g. case U4). This may happen when $R i_{*}$ is small enough. Serizawa \& Kataoka (1990) attributed this 'turbulence reduction' to a transfer of turbulence kinetic energy from the liquid to the bubble surface energy such that the resulting deformation would lead to eddy break-up and to energy dissipation increase. Although this explanation is attractive, we believe in a simpler physical mechanism. At small $R i_{*}$, the bubble-induced turbulence is small or at least much smaller than the shear-induced turbulence. But this last mechanism is less effective than in single-phase flow because the bubbles inhibit the turbulence production through the reduction of the turbulence shear stress (see figures 9 and 10). In this case $\overline{u_{L} v_{L}} / u_{*}^{2}$ and also $\overline{u_{L} v_{L}}$ are smaller in bubbly flow than in single-phase flow.

(v) The turbulence level in the core can be much larger in two-phase flow than in single-phase flow (e.g. case U1 in figure $11 b$ ). When $R i_{*}$ is large enough, (6.14) shows that $k_{L B}$ can be much larger where it is produced than $k_{L S}$. As a consequence, when $R i_{*}$ increases the core radius increases to the detriment of the inertial layer. For large enough $R i_{*}$ it may even happen that the core invades the cross-section in such a way that the inertial layer disappears (see for example figure 7 for the case U1). These cases are particularly interesting: indeed as the core turbulence mainly comes from bubble slip they can be used to verify (6.13). Assuming uncoupled mechanisms it is always possible to determine $k_{L B} \approx k_{L}-k_{L S}$ if $k_{L S}$ is known. One might estimate $k_{L S}$ through the value $k_{L 0}$ it takes in singlephase flow. But the assumption $k_{L S} \approx k_{L 0}$ would overestimate the shear-induced turbulence, as pointed out before. Hopefully there are cases for which $k_{L S}$ is small compared to $k_{L}$ so that most of the energy comes from bubble-induced turbulence $\left(k_{L B} \approx k_{L}\right)$. Equation (6.14) suggests that this assumption should be true for large enough value of $\langle\alpha\rangle U_{L G}^{2} / u_{*}^{2}$, i.e. of $R i_{*}$. The present experiments at $R i_{*}>1$ plotted in figure 12(a) show that the core turbulence is scaled well by $\alpha U_{L G}^{2}$ as the theory of (6.12) and (6.13) predicts, and that $u_{L}^{\prime 2} /\left(\alpha U_{L G}^{2}\right)$ nearly falls in the range $[1.5,2.5]$ found by Lance \& Bataille (1991).

While the turbulence kinetic energy in the wall layer possesses all the features displayed in single-phase flow that of the core is more specific. In some respects it may even appear surprising. Its signature $u_{L, a x i s}^{\prime} / u_{*}$ is plotted in figure $12(b)$. When $\sqrt{\langle\alpha\rangle} U_{L G} / u_{*}$ is greater than $\sim 0.5$, it is dominated by the bubble-induced turbulence with a rough asymptotic value of $\sqrt{2 \alpha} U_{L G} / u_{*}$. When $\sqrt{\langle\alpha\rangle} U_{L G} / u_{*}$ approaches zero, it tends to the acknowledged value of $\sim 0.8$ in single-phase flow and it slightly increases to greater values for greater $\langle\alpha\rangle$ in microgravity where a fluctuating motion of the bubbles exists. But we do not know what happens for $\sqrt{\langle\alpha\rangle} U_{L G} / u_{*}<0.5$. It is likely 

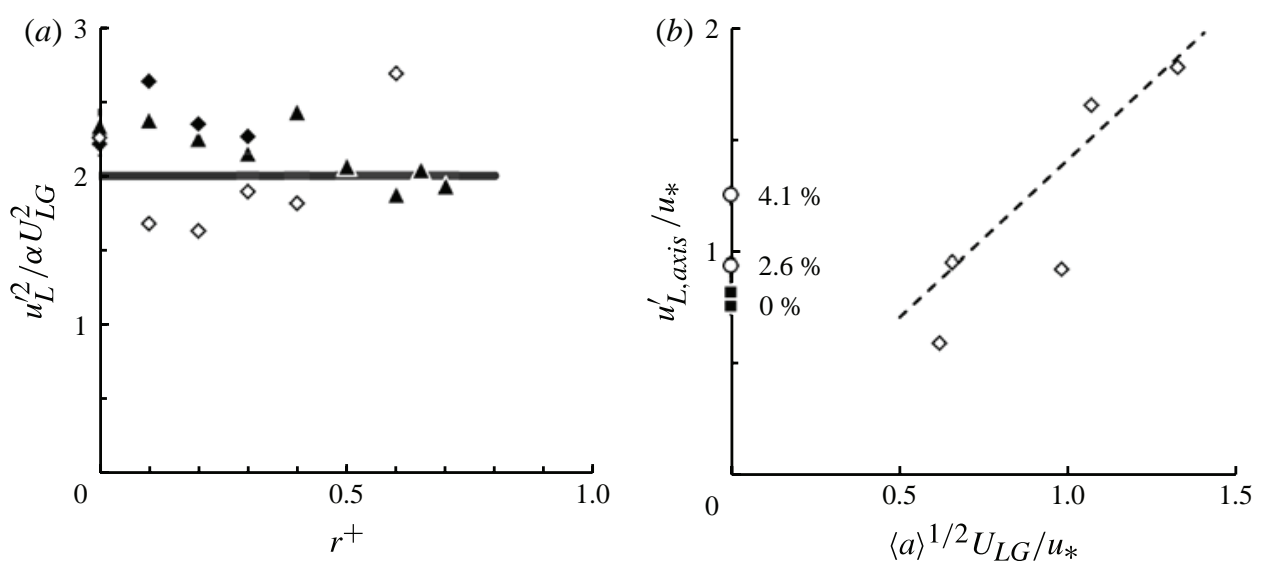

FIgURE 12. Dimensionless liquid r.m.s. velocities. (a) In the core for $R i_{*}>1: \boldsymbol{\Delta}, \mathrm{U} 1 ; \boldsymbol{\triangleleft}, \mathrm{U} 3$; $\diamond$, D3. (b) At the axis: $\mathbf{\square}$, single-phase; $\bigcirc$, microgravity; $\diamond$, upward and downward.
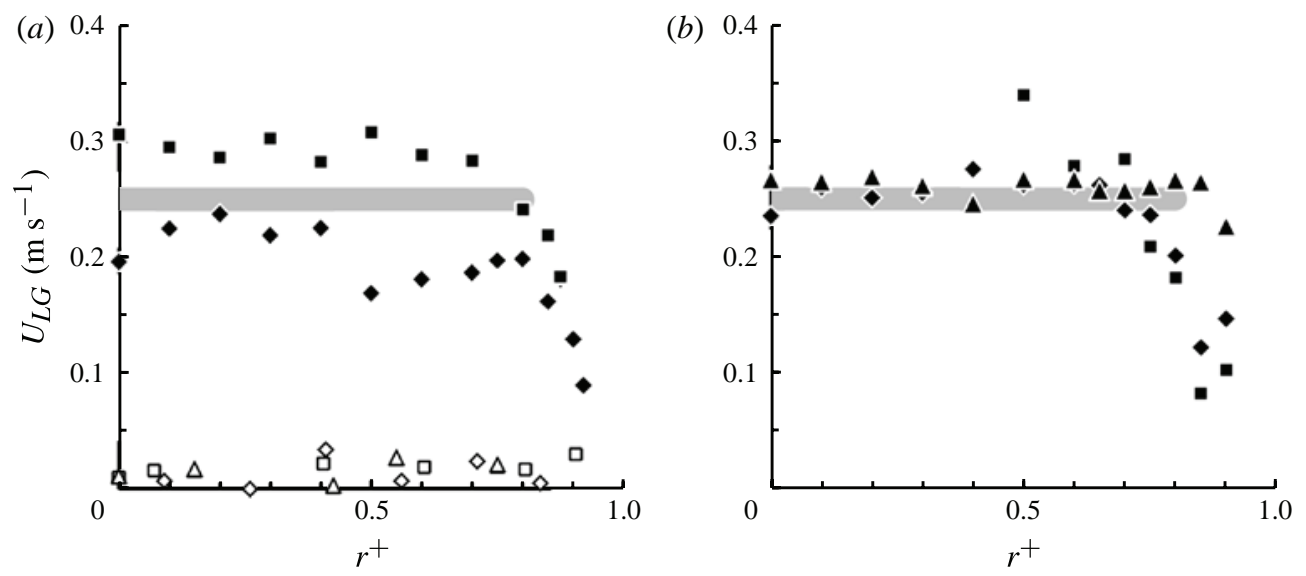

FIGURE 13. Bubble slip velocity compared to the rise velocity in still liquid from (6.16) (horizontal grey line): $(a)(\triangle), \mathrm{M} 1 ;(\diamond), \mathrm{M} 3 ;(\square), \mathrm{M} 4 ;(\diamond), \mathrm{D} 3 ;(\boldsymbol{\square}), \mathrm{D} 4 ;(b)(\mathbf{\Delta}), \mathrm{U} 1 ;(\diamond), \mathrm{U} 3$; (ם), U4.

that $u_{L, \text { axis }}^{\prime} / u_{*}$ goes through a minimum. It must be stressed that given the small number of flow conditions this graph has no predictive capability.

\subsection{Bubble slip velocity}

Buoyancy induces a drift between the two phases. The resulting slip velocity is positive in upward flow, negative in downward flow and near zero at microgravity conditions. The absolute value of the slip velocity is plotted in figure 13(a) for downward and microgravity flows and in figure 13(b) for upward flow.

The terminal velocity in still liquid $U_{\infty}$ of ellipsoidal bubbles of equivalent diameter $d$ is well-predicted, for the capillaro-gravity regime by the relation of Mendelson 
(1967):

$$
U_{\infty}=\left(2.14 \frac{\sigma}{\rho_{L} d}+0.505 \gamma d\right)^{1 / 2}, \quad d>0.44 \sqrt{\frac{\sigma}{\rho_{L} \gamma}} .
$$

The present upward or downward experiments belong to this regime since the condition is fulfilled for $d>1.2 \mathrm{~mm}$. Equation (6.16) gives a terminal velocity in still liquid of $0.26-0.24 \mathrm{~m} \mathrm{~s}^{-1}$ for bubbles of $3-4.5 \mathrm{~mm}$ equivalent diameter.

The microgravity experiments do not belong to the capillaro-gravity regime: the residual gravity in the $x$-direction is less than 0.002 times the terrestrial gravity and thus the inequality of (6.16) is not satisfied. They belong rather to the viscous regime predicted by the Hadamard-Rybczynski equation (see Clift, Grace \& Weber 1978):

$$
U_{\infty}=\frac{1}{12} \frac{\gamma d^{2}}{v}, \quad d<\left(36 \frac{v^{2}}{\gamma}\right)^{1 / 3} .
$$

For example bubbles of $1.2 \mathrm{~mm}$ diameter that satisfies the above condition would have a velocity of $U_{\infty} \approx 3.7 \mathrm{~mm} \mathrm{~s}^{-1}$ because of the residual gravity.

In the flow core we expect the slip velocity to be close to the predicted velocity in still liquid because both the velocity and the turbulence are nearly homogeneous (figure 13). This is the case at microgravity conditions (figure 13a) for which the measured slip velocity remains comparable to the value of $U_{\infty}$ for $1.2 \mathrm{~mm}$ diameter bubbles. It is also the case for upward flow for which the slip velocity is nearly that of the terminal velocity in still liquid in the capillaro-gravity regime (figure 13b). However in downward flow the difference between the measured slip velocity and the predicted velocity in still liquid is quite important (figure 13a). As the turbulence has a negligible effect on the slip velocity in the ellipsoidal bubble regime (Eppinger 1995), only a measurement bias can explain the observed difference: this is a possible explanation since the slip velocity is determined from the difference between two measured velocities whose accuracy is probably not better than $2 \%$.

In the near-wall region, the slip velocity decreases (figure 13). Even if the bubble diameter decreases as well (figures 4 and 5) their dynamics belong to the capillarogravity regime for which the velocity in still liquid depends little on the bubble diameter. Why is the slip velocity much smaller than the velocity in still liquid in the wall region? A recent numerical experiment (Adoua, Legendre \& Magnaudet 2009) has shown that the drag coefficient of an oblate bubble increases with the shear rate $S r=2 d U_{L, y} / U_{L}$ if it is greater than 0.2. For $S r=1$, they found that the drag coefficient can be more than twice its value for a homogeneous flow. In the present experiments $S r$ can increase up to 1 when the distance from the wall decreases. At this stage it is impossible to check how much the shear rate can reduce the slip velocity because the process is sensitive to the bubble aspect ratio, a quantity that has not been determined in our experiments.

\subsection{Fluctuating velocity of the gas phase}

The ratio of the gas to liquid r.m.s. velocities $u_{G}^{\prime} / u_{L}^{\prime}$ is plotted in figure 14. Its value typically ranges between 1 and 3 and decreases near the wall. It is smaller for downward flow than for upward flow and it seems to increase when the Reynolds number increases.

A theoretical expression of this ratio was first established by Tchen (1947) for particles whose density is larger or smaller than that of the carrying phase (see also Hinze 1959, pp. 357-359). The idea of Tchen was to investigate in the linear limit 

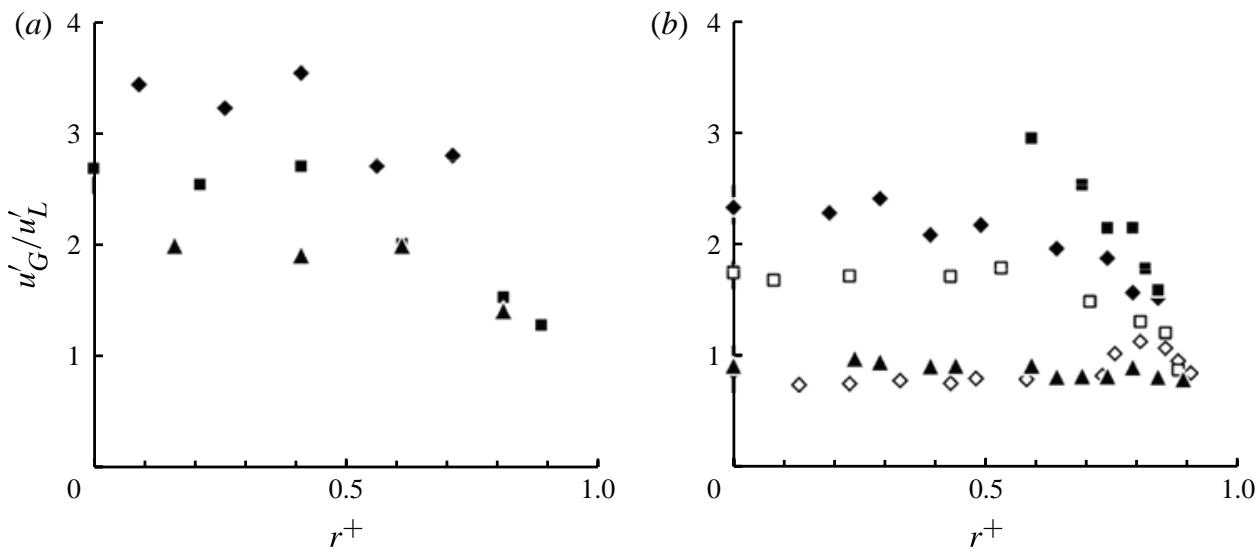

FIGURE 14. Ratio of the gas to liquid r.m.s. velocities: $(a) \mathbf{\Delta}, \mathrm{M} 1 ; \diamond, \mathrm{M} 3 ; \mathbf{\square}, \mathrm{M} 4 ;(b) \diamond, \mathrm{D} 3$; $\square, \mathrm{D} 4 ; \boldsymbol{\Delta}, \mathrm{U} 1 ; \diamond, \mathrm{U} 3 ; \mathbf{\square}, \mathrm{U} 4$.

the response of a small particle to the excitation induced by the fluctuating velocity of the carrier fluid. Under some restrictive assumptions (steady homogeneous turbulence, spherical particles smaller than the Kolmogorov microscale) the Lagrangian energy spectrum for the particle can be calculated versus that of the carrier phase. After integrating over the whole spectrum, the ratio of the r.m.s. velocity of the particle to that of the fluid is obtained:

$$
\frac{u_{G}^{\prime 2}}{u_{L}^{\prime 2}}=\frac{b^{2}-t_{L} / t_{G}}{1-t_{L} / t_{G}}, \quad b=\frac{3}{1+2 \rho_{G} / \rho_{L}} \approx 3, \quad t_{G}=\frac{\left(1+2 \rho_{G} / \rho_{L}\right) d^{2}}{24 v} \approx \frac{d^{2}}{24 v},
$$

where the above expressions are applied to bubbles moving in a continuous liquid; $t_{G}$ is the bubble relaxation time and $t_{L}$ is the Lagrangian integral time scale of turbulence. The assumptions underlying the above expressions are seldom satisfied in bubbly flow. However, the microgravity conditions seem to be a reasonable frame in which to apply the Tchen theory because the effect of the mean slip velocity on the bubble dispersion, also known as 'crossing trajectory effect' (Csanady 1964), is absent.

Some results from direct numerical simulations (Yeung \& Pope 1989) or from experiments (Sato \& Yamamoto 1987) provide a relation between $t_{L}$ and the Eulerian integral time scale $T_{e}: t_{L} \approx 0.5 T_{e}$. Moreover, near the pipe axis, the Eulerian integral time scale can be estimated versus the Eulerian length scale $l_{e}$ and the r.m.s. velocity of the liquid by $T_{e} \approx l_{e} / u_{L}^{\prime} \approx \kappa D / 2 u_{L}^{\prime}$. From these expressions, we estimated the values of the ratio $u_{G}^{\prime} / u_{L}^{\prime}$ near the pipe axis at microgravity conditions (table 3 ). These values are slightly smaller than those found in the present experiments. As shown for the runs M3 and M4 (figure 14) they are indeed close to 3, the value that would be obtained from the Tchen theory in inviscid fluid, i.e. in the absence of drag. The Tchen theory based on the Stokes drag assumption underestimates the ratio $u_{G}^{\prime} / u_{L}^{\prime}$ because this assumption is not valid when the bubble Reynolds number based on the fluctuating slip velocity is not small.

An extension of the Tchen theory to higher bubble Reynolds number has been proposed by Deutch \& Simonin (1991). It allows accounting for the crossing trajectory effect. The bubble relaxation time $t_{G}$ and the Lagrangian integral time scales of 


$\begin{array}{cccccc}\text { Run } & r^{+} & \begin{array}{c}d \\ (\mathrm{~mm})\end{array} & \begin{array}{c}u_{L}^{\prime} \\ \left(\mathrm{m} \mathrm{s}^{-1}\right)\end{array} & \begin{array}{c}u_{G}^{\prime} / u_{L}^{\prime} \\ \text { M1 }\end{array} \mathbf{v}_{G}^{\prime} / v_{L}^{\prime} \\ \text { M3 } & 0 & 2 & 0.03 & 2.3 & 2.3 \\ \text { M4 } & 0 & 1.8 & 0.06 & 2.5 & 2.5 \\ \text { U1 } & 0 & 3.2 & 0.05 & 2.1 & 2.1 \\ \text { U3 } & 0 & 3.5 & 0.07 & 1.9 & 2.2 \\ \text { U4 } & 0.6 & 3.4 & 0.046 & 1.9 & 2.2 \\ \text { D3 } & 0 & 4 & 0.096 & 1.8 & 2.7 \\ \text { D4 } & 0 & 3 & 0.053 & 2 & 2.3\end{array}$

TABLE 3. Ratio of the gas and liquid r.m.s. velocities.

turbulence in the axial and radial directions $t_{L x}$ and $t_{L r}$ can be written:

$$
t_{G}=\frac{\rho_{G} / \rho_{L}+C_{M}}{3 C_{D} U_{L G} / 4 d}=\frac{U_{L G}}{2 \gamma}, \quad t_{L x}=\frac{t_{L}}{\sqrt{1+\left(U_{L G} / 2 u_{L}^{\prime}\right)^{2}}}, \quad t_{L r}=\frac{t_{L}}{\sqrt{1+\left(U_{L G} / u_{L}^{\prime}\right)^{2}}} .
$$

The ratio of the r.m.s. velocities of the bubbles and of the liquid in the axial and radial directions are expressed versus these time scales:

$$
\frac{u_{G}^{\prime 2}}{u_{L}^{\prime 2}}=\frac{b^{2}+t_{L x} / t_{G}}{1+t_{L x} / t_{G}}, \quad \frac{v_{G}^{\prime 2}}{v_{L}^{\prime 2}}=\frac{b^{2}+t_{L r} / t_{G}}{1+t_{L r} / t_{G}} .
$$

For the runs dominated by gravity, the values of $u_{G}^{\prime} / u_{L}^{\prime}$ and $v_{G}^{\prime} / v_{L}^{\prime}$ are estimated using (6.20) at the pipe axis for U1, U3, D3, D4 and at $2 r / D=0.6$ for U4 (table 3). The values of $u_{G}^{\prime} / u_{L}^{\prime}$ are lower than in microgravity. For U4 and D4 they are in good agreement with the experimental values. When the bubble-induced turbulence plays a significant role (D3 and U1) (6.20) overestimates the experimental values which are closer to 1 . The ratio $v_{G}^{\prime} / v_{L}^{\prime}$ is typically $15 \%$ higher than $u_{G}^{\prime} / u_{L}^{\prime}$ because the ratio of the Lagrangian integral time scales of turbulence $t_{L x} / t_{L r}$ is close to 2 .

\subsection{Gas fraction}

Several studies have been devoted to the prediction of the gas fraction distribution in pipes using Eulerian two-fluid models (Drew \& Lahey 1982; Antal, Lahey \& Flaherty 1991; Lance \& Lopez de Bertodano 1994; Lopez de Bertodano et al. 1994; Chahed \& Masbernat 1998; Chahed, Colin \& Masbernat 2002; Chahed, Roig \& Masbernat 2003; Lucas et al. 2007; Hosokawa \& Tomiyama 2009b). Some of these models are used here to analyse the influence of buoyancy. They start from the radial momentum equation of each phase written for a fully developed steady flow. By assuming that the turbulence is isotropic and that the gas inertia is negligible, one obtains the differential equation that governs the gas fraction distribution by eliminating the pressure gradient between these two equations:

$$
\rho_{L} \alpha \frac{\mathrm{d}(1-\alpha) v_{L}^{\prime 2}}{\mathrm{~d} r}+M_{G r}=0,
$$

where $v_{L}^{\prime 2}$ is the radial contribution of the turbulent kinetic energy, $M_{G r}$ is the radial component of the force $M_{G}$ per unit volume exerted by the liquid on the gas bubbles. This force is expressed as a sum of three contributions: the drag, the lift and the added mass forces (e.g. Magnaudet, Rivero \& Fabre 1995). By taking into account the 
direction of each contribution, the radial component reduces to

$$
M_{G r}=-\rho_{L} \alpha C_{L} U_{L G} \frac{\mathrm{d} U_{L}}{\mathrm{~d} r}+M_{G T r},
$$

where the first term of the right-hand side is the lift force introducing the lift coefficient $C_{L}$ and the second term is the contribution of the nonlinear terms resulting from the averaging procedure. This last term includes the fluctuating contributions of the drag, the lift and the added mass force. A classical approach used by most of the authors consists in modelling the effect of turbulence by a 'turbulent dispersion' force that can be written (Lance \& Lopez de Bertodano 1994):

$$
M_{G T r}=-C_{D T} \rho_{L} k_{L S} \frac{\mathrm{d} \alpha}{\mathrm{d} r},
$$

where $C_{D T}$ is a turbulent dispersion coefficient between 0 and 1 from different expressions found in the literature.

Written in dimensionless form, (6.21) becomes

$$
\left(\alpha v_{L}^{\prime+2}+C_{D T} k_{L S}^{+}\right) \frac{\mathrm{d} \alpha}{\mathrm{d} r^{+}}=\alpha(1-\alpha) \frac{\mathrm{d} v_{L}^{\prime+2}}{\mathrm{~d} r^{+}}-\alpha C_{L} \frac{U_{L G}}{u_{*}} \frac{\mathrm{d} U_{L}^{+}}{\mathrm{d} r^{+}},
$$

where the superscript + denotes dimensionless quantities scaled with $u_{*}$ and $D / 2$. The above equation can be viewed as a differential equation in $\alpha$ once the terms of the right-hand side and thus the distributions of $U_{L}^{+}$and $v_{L}^{\prime+}$ are known. In the core region both the gas fraction and the mean velocity are almost constant so that (6.24) tells us nothing more than we already know. In the near-wall region this is not the case since all the physical quantities including $U_{L}^{+}$an $v_{L}^{\prime+}$ present steep gradients: the first term of the right-hand side is positive and the second one, the lift force, is positive in upward flow and negative in downward flow. Because their ratio $U_{L G} / u_{*}$ is $O(1)$ in that region (in fact $U_{L G} / u_{*} \approx 5$ in our experiments), it controls the bubble distribution through the lift force. For bubbles smaller than $\sim 5 \mathrm{~mm}$, the lift coefficient is positive according to Tomiyama et al. (2002) and the lift force tends to push the bubbles toward the wall in upward flow and toward the axis in downward flow. To avoid an accumulation of bubbles at the wall in upward flow, most authors introduce a wall force (Antal et al. 1991) that artificially fixes the location of the maximum gas fraction and leads to a negative gradient of $\alpha$ near the wall. We verified that for gravity flows such a model predicts the gas fraction distributions satisfactorily. But at microgravity conditions this model is not in agreement with our current observations. Because $U_{L G} / u_{*}$ is very small the lift becomes negligible and the sign of $\mathrm{d} \alpha^{+} / \mathrm{d} r^{+}$is the same as that of $\mathrm{d} v_{L}^{\prime+2} / \mathrm{d} r^{+}$. This is obviously untrue since the experiments confirm that $\mathrm{d} \alpha^{+} / \mathrm{d} r^{+}$is negative while $\mathrm{d} u_{L}^{\prime+2} / \mathrm{d} r^{+}$and thus $\mathrm{d} v_{L}^{\prime+2} / \mathrm{d} r^{+}$are positive. Special attention has to be paid to the modelling of the turbulent terms in the interfacial momentum transfer especially in microgravity conditions. We note in passing that microgravity flow is an interesting test for understanding the influence of these nonlinear terms.

From theoretical arguments Chahed \& Masbernat (1998) and Chahed et al. (2002) proposed modelling the nonlinear terms resulting from averaging the drag and the added mass forces:

$$
M_{G T r}=\frac{3}{4} \frac{C_{D}}{d}\left|U_{L G}\right| V_{d}-\frac{C_{M}}{r} \frac{\mathrm{d}}{\mathrm{d} r}\left[r \alpha\left(v_{G}^{\prime 2}-v_{L}^{\prime 2}\right)\right],
$$


where $C_{M}$ is the added mass coefficient, $C_{D}$ is the drag coefficient and $V_{d}$ is a radial drift velocity. This velocity is modelled as a dispersion term proportional to the gas fraction gradient and the eddy diffusivity $v_{t}$ as $V_{d}=-C_{D T} v_{t} \mathrm{~d} \alpha / \mathrm{d} r$, where $C_{D T}$ is a constant coefficient. With this model, (6.21) becomes in a dimensionless form

$$
\begin{aligned}
& {\left[\alpha v_{G}^{\prime+2}+C_{M}\left(v_{G}^{\prime+2}-v_{L}^{\prime+2}\right)-\frac{3}{4} C_{D} C_{D T} C_{\mu} v_{t}^{+} \frac{U_{L G}}{u_{*}}\right] \frac{\mathrm{d} \alpha}{\mathrm{d} r^{+}}} \\
& \quad=\alpha(1-\alpha) \frac{\mathrm{d} v_{L}^{\prime+2}}{\mathrm{~d} r^{+}}-\alpha C_{L} \frac{U_{L G}}{u_{*}} \frac{\mathrm{d} U_{L}^{+}}{\mathrm{d} r^{+}}-C_{M} \frac{\alpha}{r^{+}} \frac{\mathrm{d}}{\mathrm{d} r^{+}}\left[r^{+}\left(v_{G}^{\prime+2}-v_{L}^{\prime+2}\right)\right],
\end{aligned}
$$

where the turbulent viscosity is scaled by $C_{\mu} u_{*} D$. While this differential equation contains some extra terms compared to (6.24), it does not include an additional dimensionless number. Indeed, both have solutions that depend on $U_{L G} / u_{*}$.

To transform (6.26) into a workable equation, we need to drop some terms and to approximate some others. The square-bracketed term of the left-hand side can be simplified as follows. The ratio of the third to the second term of the left-hand side is $\sim 0.2$ in the capillaro-gravity regime and 0.005 in the viscous regime (this evaluation was done with $C_{D T}=0.35, C_{\mu}=0.09$ and $C_{D}=0.3 \rho_{L} U_{L G}^{2} d / \sigma$ for the capillaro-gravity regime and $16 v /\left(U_{L G} d\right)$ for the viscous regime). The third term will be ignored. Because $\left(v_{G}^{\prime+2}-v_{L}^{\prime 2}\right)$ is of the order of $\left(v_{L}^{\prime+2}\right)$, the first term of the left-hand side will be ignored at small gas fraction. And for the same reason, $(1-\alpha)$ is about 1 in the first term of the right-hand side. Equation (6.26) has the same shape as (6.24), the second terms in the bracket of the right-hand sides of these two equations being of the order of $k_{L S}^{+}$. In normal gravity, the dominant term is the lift force (second term of the right-hand side) while at microgravity it is zero, the two other terms becoming dominant.

By simplifying (6.26), we get a differential equation in $\alpha$ that is valid in the wall layer $\left(r_{c}^{+}<r^{+}<1\right.$, where $\left.r_{c}^{+}=1-\delta^{+}\right)$where the log-law for the mean velocity profile is valid and where the turbulent kinetic energy can be estimated by a balance between its production and its dissipation. Moreover, in this region, the turbulent shear stress is almost piecewise linear (figure 9):

$$
\overline{u^{+} v^{+}}=a r^{+}+b, \quad a=\frac{1-c r_{c}^{+}}{1-r_{c}^{+}}, \quad b=\frac{(c-1) r_{c}^{+}}{1-r_{c}^{+}} .
$$

In this equation $c$ is in the range [0,1]: 0 when the shear stress is zero in the core and 1 when it is linear, i.e. in microgravity. In addition, the following approximations for the mean velocity gradient and the turbulent terms are used:

$$
\frac{\mathrm{d} U_{L}^{+}}{\mathrm{d} r^{+}}=-\frac{1}{\kappa\left(1-r^{+}\right)}, \quad v_{L}^{\prime+2}=\frac{2}{3} k_{L S}^{+}, \quad \frac{v_{G}^{\prime}}{v_{L}^{\prime}}=C_{t}, \quad k_{L S}^{+}=\frac{\overline{u^{+} v^{+}}}{\sqrt{C_{\mu}}},
$$

where $C_{t}$ is a constant greater than unity (Eppinger 1995).

With these assumptions, (6.26) becomes

$$
\frac{\mathrm{d} \alpha}{\alpha}=\left[\frac{a\left(C_{0}-1\right)}{a r^{+}+b}-\frac{1}{r^{+}}+\frac{C_{g}}{\left(1-r^{+}\right)\left(a r^{+}+b\right)} \frac{U_{L G}}{u_{*}}\right] \mathrm{d} r^{+},
$$

with

$$
C_{0}=\frac{1}{C_{M}\left(C_{t}^{2}-1\right)}, \quad C_{g}=\frac{3 C_{L} \sqrt{C_{\mu}}}{2 \kappa C_{M}\left(C_{t}^{2}-1\right)} .
$$


(a)

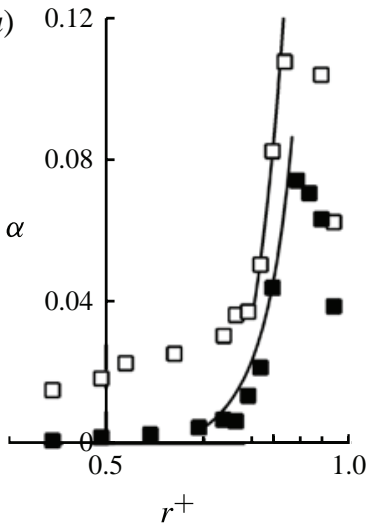

(b)

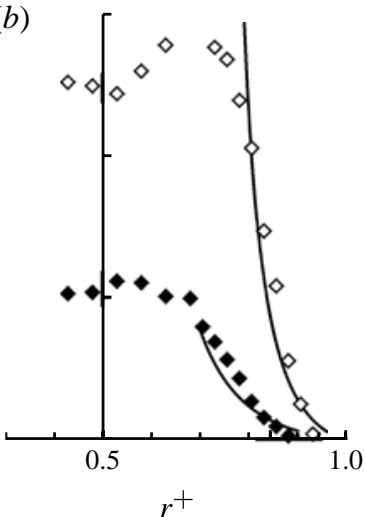

(c)

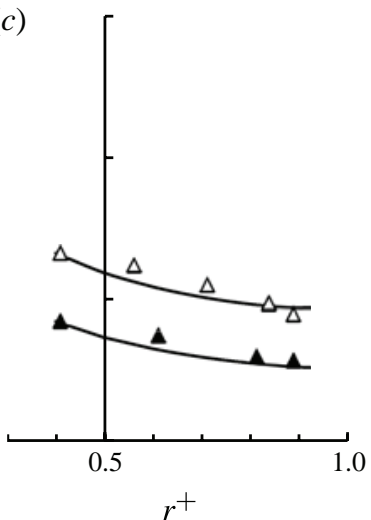

FIGURE 15. Gas fraction distribution: (a) Upward flow U3 ( $\square$ ), U4 (ם); (b) Downward flow D3 $(\diamond)$, D4 $(\diamond)$; $(c)$ Microgravity flow M3, $(\triangle)$ M4 ( $\mathbf{\Delta})$. Lines are calculations from (6.31).

Integrating (6.29) leads to the general solution of the gas fraction distribution in the wall layer, i.e. for $r^{+}>r_{c}^{+}$,

$$
\frac{\alpha}{\alpha_{c}}=\frac{f\left(r^{+}\right)}{f\left(r_{c}^{+}\right)}, \quad f\left(r^{+}\right)=\frac{\left(a r^{+}+b\right)^{\left(C_{0}-1\right)}}{r^{+}}\left(\frac{a r^{+}+b}{1-r^{+}}\right)^{C_{g} U_{L G} / u_{*}} .
$$

Note that the wall layer concept does not apply in microgravity. In that case, $\alpha_{c}$ is simply the value of $\alpha$ for an arbitrary location $r_{c}^{+}$: it can be 0 or any other value chosen in the range $[0,1]$. For gravity flow the radius of the core is deduced from the thickness of the wall layer as $r_{c}^{+}=1-\delta^{+}$. We took for $\delta^{+}$and $c$ the experimental values. In addition we did not include the wall force in our radial momentum balance so that the gas fraction profile cannot be calculated up to the wall in upward flow.

The solution for $\alpha$ is obtained once the various constants are given. The lift coefficient has a major effect on the radial distribution of $\alpha$ : we chose $C_{L}=0.288$ according to Tomiyama et al. (2002). In the experiments, the aspect ratio of the bubbles is close to 1.5: according to Lamb (1932) the added mass coefficient for an ellipsoidal bubble with an aspect ratio of 1.5 moving perpendicularly to its minor axis is $C_{M} \approx 0.4$. The values of $u_{G}^{\prime} / u_{L}^{\prime}$ in the near-wall region are a little smaller than the theoretical values given by (6.20) and table 3 . It is thus expected that the values of $C_{t}=v_{G}^{\prime} / v_{L}^{\prime}$ would also be smaller, say between 1.5 and 2 : we took $C_{t}=1.8$.

Figure 15 displays a comparison between the calculated and the measured values of $\alpha$. As the solution (6.31) has been developed for shear-induced turbulence, its validity is limited to the wall region where the log-law applies. In that region the agreement between the analytical solution and the experiments is quite good. But it cannot fit the trends very near the wall and in the core where the turbulence modelling that has been used here is failing. Despite the relatively simple and perhaps incomplete formulation of the nonlinear terms in the interfacial momentum transfer, this simple analytical model gives a good idea of the gas fraction distribution in various gravity conditions. Even if it cannot be considered as a predictive tool it has the merit of highlighting the following points.

(i) The nonlinear terms in the interfacial momentum transfer play a special role in the gas fraction distribution. These terms are of the same order of magnitude as the lift force in upward and downward flows. They become crucial in microgravity when 
the lift force disappears. In this configuration the contribution of the turbulence added mass force seems to be dominant. This contribution could not emerge without the microgravity experiments.

(ii) The gas fraction distribution near the wall depends on the dimensionless parameter $U_{L G} / u_{*}$ that quantifies the effect of buoyancy versus friction.

Realistic progress in the modelling of the interfacial momentum transfer could be expected from experiments and numerical simulation of Lagrangian tracking of isolated bubbles in turbulent shear flows. Some experiments (Colin \& Legendre 2001) and numerical simulations (Legendre et al. 1999) on the motion of isolated bubbles in a turbulent pipe flow confirmed that, on the Earth, the bubbles move towards the wall in vertical upward flow and towards the centre in downward flow. In microgravity where the lift force is negligible, the action of the large turbulent eddies on bubble dispersion is dominant and the radial bubble distribution is controlled by the temporal variations of the turbulent structures.

\subsection{Wall friction}

In the previous subsection we have shown that the radial distributions of velocity, turbulence and gas fraction are sensitive to gravity and wall friction through various dimensionless parameters:

(i) the liquid velocity and the turbulent shear stress depend on $R i_{*}$;

(ii) the turbulence in the core region is sensitive to $\alpha^{1 / 2} U_{L G} / u_{*}$;

(iii) the gas fraction in the wall region is controlled by $U_{L G} / u_{*}$.

All these dimensionless numbers relate the buoyancy and friction effects, directly or indirectly. However, though for bubbles in a viscous regime $U_{L G}$ is related to $\gamma d$ through a rather simple relation, as seen in (6.17), this is not the case in capillarogravity regime as (6.16) shows. Because most of the experimental results have been obtained for this regime, it may explain why the literature does not provide a clear answer to the scaling of buoyancy to friction effects in bubbly flow.

To predict what the flow behaviour looks like it is important to evaluate these dimensionless numbers, i.e. to estimate $\langle\alpha\rangle, U_{L G}$ and $u_{*}$. The first two quantities can be easily determined for given bubble diameter and volumetric quality $\chi=j_{G} /\left(j_{L}+j_{G}\right)$. In contrast the evaluation of $u_{*}$ is more problematic. Little has been done in the previous studies to predict $u_{*}$, i.e. the wall shear stress $\tau_{w}$. Indeed in vertical bubbly flow the wall friction has a small influence on the pressure gradient. This is due to the fact that $4 \tau_{w} / D$ is usually small compared to the weight of the mixture. As a consequence the prediction of the pressure gradient does not require that the wall shear stress $\tau_{w}$ be known with a great accuracy. This is likely to be why it has been disregarded.

Marié et al. (1997) were perhaps the only ones to develop a theoretical model for the wall shear stress. The model was tested against their data in an upward bubbly boundary layer. It was also tested against downward pipe flow data. In their model the friction velocity is one of the roots of a quadratic equation. We put this solution into the form

$$
\frac{u_{*}}{u_{* 0}}=\frac{\left(1-C \sqrt{f_{0} / 2}\right) \sqrt{1+\left(1-2 C \sqrt{f_{0} / 2}\right) R i_{* d}}-C \sqrt{f_{0} / 2}}{1-2 C \sqrt{f_{0} / 2}},
$$

where $C=10.6, u_{* 0}$ is the friction velocity in the single-phase flow of the same liquid velocity $\left\langle U_{L}\right\rangle=j_{L} /(1-\langle\alpha\rangle), f_{0}=2\left(u_{* 0} /\left\langle U_{L}\right\rangle\right)^{2}$ is the corresponding friction factor 
and $R i_{* d}$ a buoyancy parameter similar to $R i_{*}$ except that it is scaled by the bubble diameter rather than by the pipe diameter and that it is weighted by the difference of gas fraction at the wall and at the core $\left(\alpha_{w}-\alpha_{c}\right)$ and not by $\langle\alpha\rangle$ :

$$
R i_{* d}=\frac{\left(\alpha_{w}-\alpha_{c}\right) \gamma d}{u_{* 0}^{2}} .
$$

It must be pointed out that, if the Reynolds number is not large enough, there exists a critical $R i_{* d}$ above which (6.32) has no real root.

To understand how the ratio $u_{*} / u_{* 0}$ depends on $R i_{* d}$ it is useful to consider the following simplified explicit form of (6.32) that is valid with a reasonable accuracy for $0<R i_{* d}<1$ :

$$
\frac{u_{*}}{u_{* 0}}=1+\frac{1}{2}\left(1-C \sqrt{f_{0} / 2}\right) R i_{* d} .
$$

Since the single-phase friction factor, $f_{0}$, is smaller than 0.018 for turbulent flow the bracketed term of the right-hand side of (6.34) is positive. Furthermore it appears that $R i_{* d}$ is always positive: this is obviously true for upward flow because $\gamma>0$ and $\alpha_{w}>\alpha_{c}$ but this is also true for downward flow since if $\gamma<0, \alpha_{w}$ is nearly zero. The relation predicts that the ratio $u_{*} / u_{* 0}$ increases with the gravity parameter $R i_{* d}$. Thus according to (6.34) the two-phase friction velocity is always greater than that of the single-phase flow of the same liquid velocity.

The present results of $u_{*}$ are compared to the friction velocity predicted by (6.32) in figure 16(a). Though it is hard to assess the model, because of the experimental uncertainty on $u_{*}$ and on $\left(\alpha_{w}-\alpha_{c}\right)$, at least the predictions are in qualitative agreement with the experimental results.

The model of (6.32), or of its simplified form (6.34), is based on a two-layer flow, a wall layer and a core layer. As such it is not fully predictive since $\alpha_{w}$ and $\alpha_{c}$ must be known. However (6.34) can be easily simplified to yield a workable correlation. In a first step $\left(\alpha_{w}-\alpha_{c}\right)$ may be approximated by $\langle\alpha\rangle$, so that (6.34) becomes

$$
\frac{u_{*}}{u_{* 0}}=\left(\frac{\tau_{w}}{\tau_{w 0}}\right)^{1 / 2}=1+\frac{1}{2}\left(1-C \sqrt{f_{0} / 2}\right) \frac{\langle\alpha\rangle|\gamma| d}{u_{* 0}^{2}},
$$

where $\tau_{w 0}$ is the wall shear stress of the single-phase flow of velocity $\left\langle U_{L}\right\rangle$. By comparing to our results and to those of Kashinsky \& Randin (1999) it appears that (6.35) works well with $C=9.5$. It can be further simplified because $f_{0}^{1 / 2}$ varies little $\left(f_{0} \propto R e^{-1 / 4}\right):$

$$
\frac{u_{*}}{u_{* 0}}=\left(\frac{\tau_{w}}{\tau_{w 0}}\right)^{1 / 2} \approx 1+\frac{1}{4} \frac{\langle\alpha\rangle|\gamma| d}{u_{* 0}^{2}}
$$

Equation (6.36) gives the wall shear stress $\tau_{w}$ as a product of two terms: the singlephase wall shear stress that depends on the Reynolds number and a corrective term that is a function of the buoyancy parameter $\langle\alpha\rangle|\gamma| d / u_{* 0}^{2}$. By doing so the effect of viscosity and that of gravity are clearly identified.

As shown in figure $16(b),(6.36)$ works equally well for upward flow and downward flow. Moreover for microgravity flow it is consistent with the suggestion of Colin, Fabre \& McQuillen (1996) who showed that the wall shear stress is given by a classical single-phase correlation using the physical properties of the liquid and the velocity of the mixture: because of the absence of drift, the mean liquid velocity $\left\langle U_{L}\right\rangle$ 

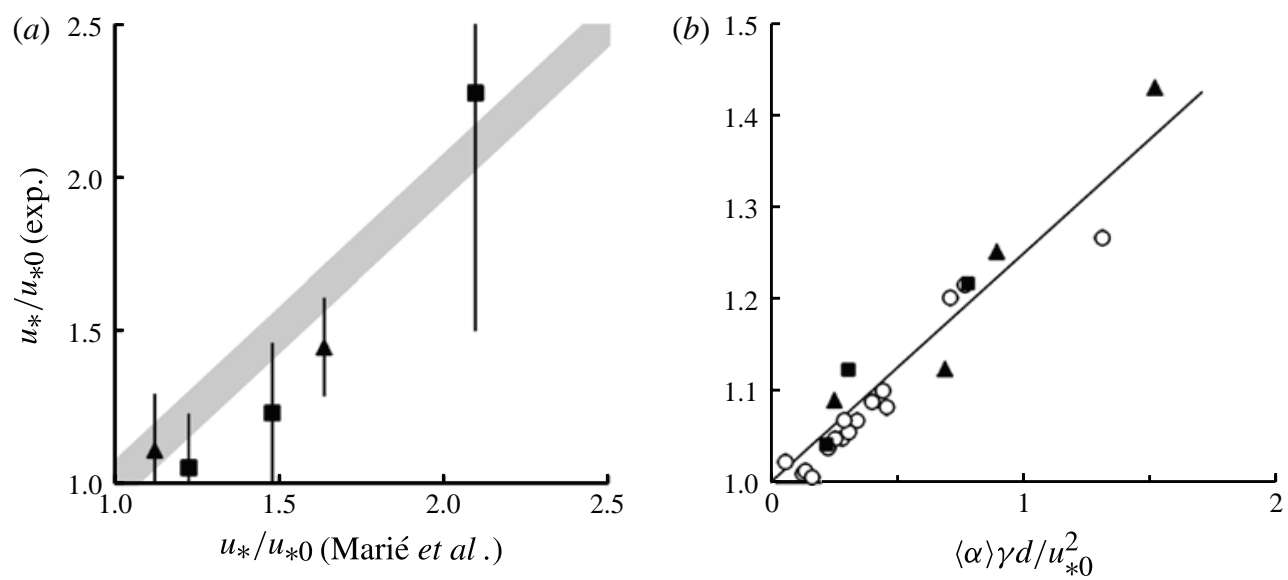

FIGURE 16. Two-phase to single-phase friction velocity. (a) Comparison with equation (6.32) (grey line): ( $\boldsymbol{\square})$, upward flow; $(\boldsymbol{\Delta})$, downward flow. (b) Comparison with equation (6.36) (-): (ם), upward flow; (ム), downward flow; (O), Kashinsky \& Randin (1999).

is equal to the mixture velocity and their correlation is obviously equivalent to the present one.

Last but not least (6.36) works for different bubble sizes (present study: 3-4 mm; Kashinsky \& Randin (1999) study: 0.8-1.7 mm). Because our and their experiments have been done with the same pipe diameter but different bubble diameter, it proves that $\sqrt{\gamma d}$ is a better velocity scale than $\sqrt{\gamma D}$ since a correlation using the latter would not fit both data sets.

\section{Conclusion}

In vertical turbulent bubbly flow, gravity plays, through bubble buoyancy, an important role that we have tried to understand and quantify through a set of experiments. These experiments were performed in the same facility with millimetric bubbles under three gravity conditions: upward, downward and microgravity flows $(1 g,-1 g$ and $0 g)$. As the flow is also controlled by friction we chose to run these experiments at the following Reynolds number and volumetric quality $\chi$ :

(i) At $\operatorname{Re} \approx 4 \times 10^{4}$ and $\chi \approx 2.5 \%$, the liquid flow rate is high and the gas fraction is small: the turbulence is mainly produced by the shear stress.

(ii) At $R e \approx 10^{4}$ and $\chi \approx 10 \%$, the liquid flow rate is low and the gas fraction is large: the turbulence induced by the bubble slip is higher than the turbulence produced by the shear stress.

(iii) At $\operatorname{Re} \approx 3 \times 10^{4}$ and $\chi \approx 6 \%$, the contributions of both the shear-induced turbulence and the bubble-induced turbulence are significant.

For each run, in addition to the flow conditions, i.e. flow rates and pressure gradient, the distributions of mean and fluctuating phase velocity, shear stress, turbulence production, gas fraction and bubble size were measured or determined. From the dimensional results the most significant effect of gravity concerns the gas fraction distribution: a wall peaking effect in upward flow and void coring in downward flow in agreement with previous studies. In microgravity, the present results show that the 
gas fraction profile is flatter than in downward flow with a maximum at the pipe axis.

To quantify the relative contributions of buoyancy and friction to the kinematic structure and the phase distribution we introduced the buoyancy number $R i_{*}$, defined in (2.2), which emerges from the momentum equation averaged over the pipe crosssection. Various physical models that have been tested against the present data reveal other buoyancy numbers $U_{L G} / u_{*}$, or $\sqrt{\langle\alpha\rangle} U_{L G} / u_{*}$, and $\sqrt{\langle\alpha\rangle}|\gamma| d / u_{* 0}^{2}$. In the present study $R i_{*}, U_{L G} / u_{*}$ and $\sqrt{\langle\alpha\rangle}|\gamma| d / u_{* 0}^{2}$ extended over the ranges [0,2.7], [0,6.4] and $[0,4.7]$ respectively.

Single-phase and microgravity flows are both insensitive to gravity $\left(R i_{*}=0\right)$ and hence should possess the same kinematic structure. Indeed, the most important features of fully developed single-phase flow are preserved in $0 g$ flow: the mean liquid velocity follows the log-law (figure 7) and the shear stress distribution is linear (figure 9). As a consequence the turbulence production in the liquid is similar and leads to the same distribution of the turbulent velocity. Concerning the gas phase we found that the bubble slip is smaller than that possibly induced by the residual gravity. Moreover the liquid-gas r.m.s. velocity ratio is around 3 in agreement with the Tchen theory. Therefore microgravity flow can be considered as a reference condition from which buoyant flows deviate.

And buoyant two-phase flows $\left(R i_{*}>0\right)$ do indeed deviate from single-phase flow. Their kinematic structure is characterized by the existence of two regions, a core and a wall layer.

(i) In the core, the phases and their mean velocity are uniformly distributed. Accordingly the absence of velocity gradient induces a severe reduction of the shear stress and of the turbulence induced by it (figure 9). Thus the core turbulence comes partly from the diffusion of the shear-induced turbulence and partly from the bubble-induced turbulence. The present results show that one or the other dominates depending on whether the buoyancy number $\sqrt{\langle\alpha\rangle} U_{L G} / u_{*}$ is less or greater than 0.5 (figure 12). Concerning the gas phase, the slip velocity is nearly that of a bubble of the same equivalent diameter in still liquid (figure 13). Moreover the liquid-gas r.m.s. velocity ratio is smaller than 3 and decreases as $R i_{*}$ increases due to the bubble slip velocity (crossing trajectory effect).

(ii) The wall layer is dominated by the shear-induced turbulence. Thus it was not surprising to find that the log-law still applies in that layer, at least under certain conditions. In the present study we found that, when $R i_{*}<1$, the log-law is valid as it is, i.e. with the same constants as those acknowledged in single-phase flow (figure 7). When $R i_{*}>1$, one can hardly say that the log-law is valid because of the sparse data available within the layer. However this law can still be used to predict the liquid velocity on the condition of the von Kármán constant $\kappa$ keeping the value it has in single-phase flow, and for the additive constant $B$ a decreasing function of $R i_{*}$ (figure 9).

The two-layer concept is not new but the fact that various laws or behaviours hold equally for upward, downward and microgravity flows is an important issue from which other properties arise. The first one concerns the thickness of the wall layer $\delta$ that results from the equilibrium between the shear- and the bubble-induced turbulence (equation (6.2)) rather than from the bubble size as previous studies suggested. The second one concerns the wall shear stress $\tau_{w}$. It is shown that the ratio $\tau_{w} / \tau_{w 0}$ can be expressed through (6.36) versus the buoyancy parameter $\sqrt{\langle\alpha\rangle}|\gamma| d / u_{* 0}^{2}$, regardless of whether the flow is upward or downward. 
The central issue of bubbly flow is the phase distribution. Its prediction involves complex numerical simulations that were outside the scope of the present study. However we selected the main mechanisms (turbulent dispersion, lift and nonlinear effects of added mass) and we tried to model them as simply as possible. The $0 g$ results are invaluable in quantifying the turbulent dispersion versus the added mass effect, while in $1 g$ or $-1 g$ flow the lift force dominates. We ended up with an analytical solution whose merit is to give a qualitative description of $\alpha$ in the wall layer and to demonstrate the role of the buoyancy number $U_{L G} / u_{*}$ in the solution that applies equally to upward, downward or microgravity flow, except very close to the wall.

\section{Acknowledgements}

We are grateful to the 'Centre National d'Etudes Spatiales' for the grant and the participation to the parabolic flight program in the 'Caravelle $0 g$ '. A part of the parabolic flights were supported by the 'European Space Agency' that we would like to thank.

\section{Supplementary data}

Supplementary data are available at journals.cambridge.org/flm.

\section{Appendix}

\section{A.1. Chord length distribution and bubble diameter distribution}

The gas fraction is assumed to be locally homogeneous over a circular section of diameter $d$ around the probe. Let the bubble size distribution which intersect the probe be given by the probability density function (p.d.f.) $P(d)$. This distribution is not equal to the distribution of the bubble sizes in this section, $P_{s}(d)$, because the larger the bubble size, the greater the number of bubbles that intersects the probe. These two distributions are related by a weighting function proportional to $d^{2}$ :

$$
P(d)=P_{s}(d) d^{2} / \int_{0}^{d_{\max }} P_{s}(d) d^{2} \mathrm{~d} d .
$$

Consider a bubble of diameter $d$ : it intersects the probe if its centre is at a distance $r$ from the probe, $r$ being smaller than $d / 2$. We assume that the bubble centres are uniformly distributed in a cylinder of diameter $d$ around the probe. After a long time, the number of bubble centres passing through an annulus of radius $r$ and thickness $d r$, is proportional to $r$. The p.d.f. of the bubble diameter with a centre at a distance $r$ from the probe and pierced by the probe tip is $P(r ; d)$. Since $P(r ; d)$ is proportional to $r$ and its integral equal to 1 , it follows that

$$
P(r ; d)= \begin{cases}8 r / d^{2}, & 0 \leqslant r \leqslant d / 2 \\ 0, & r>d / 2 .\end{cases}
$$

When a bubble touches the probe tip at a distance $r$ from its centre, the chord length $y$ intersected by the probe in the direction of a minor axis of the bubble can be calculated from

$$
r^{2}+\left(\frac{y}{2 E}\right)^{2}=\left(\frac{d}{2}\right)^{2},
$$


where $E$ is the bubble aspect ratio. We assume that the minor axis is aligned with the direction of the probe. The probability of measuring a chord with a length $y$ is

$$
P(y ; d)|\mathrm{d} y|=P(r ; d)|\mathrm{d} r| .
$$

It can be calculated using (A 3) and (A 4):

$$
P(y ; d)=\frac{2 y}{(E d)^{2}} .
$$

The p.d.f. of the chord lengths intersected by the probe becomes

$$
P(y)=P(y ; d) P(d)=\frac{2 y}{(E d)^{2}} P(d) .
$$

The backward transformation of Clark \& Turton (1988) allows the determination of the p.d.f. of bubble sizes $P(d)$ versus the measured p.d.f. of the chords lengths $P(y)$. Since the bubbles were assumed to move in a direction parallel to their minor axis, the maximum chord length $y_{\max }$ of the distribution was obtained when the probe tip pierces the bubble with the largest size $d_{\max }$ along its minor axis:

$$
y_{\max }=E\left(d_{\max }\right) d_{\max } \text {. }
$$

Then, the p.d.f. $P(d)$ and $P(y)$ were divided into $m$ classes of equal length:

$$
\text { for } 0 \leqslant i \leqslant m \quad\left\{\begin{array}{l}
d_{i}=d_{\max }(1-i / m) \\
y_{i}=E_{i} d_{i} .
\end{array}\right.
$$

By using (A 8), the mean chord length $W_{i}$ inside one class can be written as

$$
W_{i}=\int_{y i+1}^{y_{i}} P(y) \mathrm{d} y=\int_{y i+1}^{y_{i}} \mathrm{~d} y \int_{0}^{d_{\max }} P(y ; d) P(d) \mathrm{d} d \approx \Delta d \sum_{j=0}^{m-1} C_{i j} P\left(d_{j}\right),
$$

where $\Delta=d_{\max } / m$ and

$$
C_{i j}=\int_{y_{i+1}}^{y_{i}} \frac{2 y}{\left(E_{j} d_{j}\right)^{2}} \mathrm{~d} y=\frac{y_{i}^{2}-y_{i+1}^{2}}{\left(E_{j} d_{j}\right)^{2}} \quad \text { for } 1 \leqslant i \leqslant m-1, j \leqslant i
$$

and

$$
C_{00}=\frac{1}{2} \int_{y_{1}}^{y_{0}} \frac{2 y}{\left(E_{0} d_{0}\right)^{2}} \mathrm{~d} y=\frac{y_{0}^{2}-y_{1}^{2}}{\left(E_{0} d_{0}\right)^{2}} .
$$

$C_{i j}$ is equal to zero for $j>i$, because a diameter $d_{j}$ can only give a chord with a length $y$ smaller than $y_{j} . C_{i j}$ is a triangular matrix and the p.d.f. of the bubble size $P(d)$ can easily be calculated in a discretized form:

$$
\begin{gathered}
P_{0}=\frac{W_{0}}{C_{00} \Delta d}, \\
P_{i}=\frac{1}{C_{i j}}\left(W_{i} \Delta d-\sum_{j=0}^{i-1} C_{i j} P_{j}\right) \quad \text { for } i>1 .
\end{gathered}
$$

The main drawback of this method comes from the cumulative errors in the calculation of $P_{i}$ from (A 11). In order to obtain an accurate bubble size distribution a great number ( $>2000$ ) of chord lengths is required to have a smooth distribution. 
This number of chord lengths can be reduced to $\sim 500$ by assuming that the probability density function of the bubble size is well-fitted by a given law. In many experimental situations and especially in the present study the bubble size distributions were wellfitted by a log-normal law (Kamp 1996):

$$
P_{s}(d)=\frac{1}{\sqrt{2 \pi} \hat{\sigma} d} \exp \left[-\frac{\log ^{2}(d / \mu)}{2 \hat{\sigma}^{2}}\right],
$$

where $\mu$ is the median diameter of the distribution and $\hat{\sigma}$ is a parameter characteristic of the width of the bubble size distribution. The p.d.f. of diameter $d$ of the bubbles pierced by the probe was calculated from (A 1):

$$
P_{s}(d)=\frac{d}{\sqrt{2 \pi} \hat{\sigma} \mu^{2}} \exp \left[-2 \hat{\sigma}^{2}-\frac{\log ^{2}(d / \mu)}{2 \hat{\sigma}^{2}}\right] .
$$

The determination of the bubble size distribution was reduced to finding $\mu$ and $\hat{\sigma}$ that minimizes

$$
\sum_{i=0}^{m-1}\left[W_{i}-\Delta d \sum_{j=0}^{m-1} C_{i j} P_{j}(\mu, \hat{\sigma})\right]^{2} .
$$

This minimization was solved by the method of Powell (1964) that allows for the minimization of functions depending on several variables.

The chord length distributions $W(y)$ measured with the optical-fibre probe are plotted in figure 17 for two different flow conditions. The number of chord lengths $N$ used for the conversion was lower than 500 and negative values in the bubble size distribution were found. They correspond to error amplifications for the small bubble sizes. In this figure the log-normal law fitting the bubble size distribution is also plotted (solid line). The two parameters of the log-normal law $\mu$ and $\hat{\sigma}$, were determined by minimizing the difference between the measured chord length distribution and the solid line (figure 17). The solid line represents the chord length distributions $W(y)$ calculated by assuming that the bubble size distributions are fitted by log-normal laws. This method provided satisfying results with a reduced number of samples. In particular it was very interesting when the gas fraction and the measurement time were small.

\section{A.2. Bubble deformation}

In the conversion of the chord length distribution into bubble size distribution, the shape of the bubbles must be given. In the range of bubble size encountered in our experiments the bubbles can be considered as spherical in microgravity and ellipsoidal in terrestrial gravity. For the estimation of the bubble aspect ratio $E(d)$, the correlation of Wellek et al. (see Clift et al. 1978, p. 181) obtained for bubbles rising in a quiescent liquid was used:

$$
E(d)=\frac{1}{1+0.163 B o^{0.757}} \quad \text { for } B o<40 \text { and } M o<10^{-6},
$$

where $B o$ is the Bond number and $M o$ is the Morton number based on the liquid properties and on the equivalent bubble diameter $d_{e q}$ :

$$
B o=\frac{\rho_{L}|\gamma| d_{e q}^{2}}{\sigma}, \quad M o=\frac{|\gamma| \rho_{L}^{3} \nu^{4}}{\sigma^{3}}, \quad d_{e q}=E^{1 / 3} d .
$$



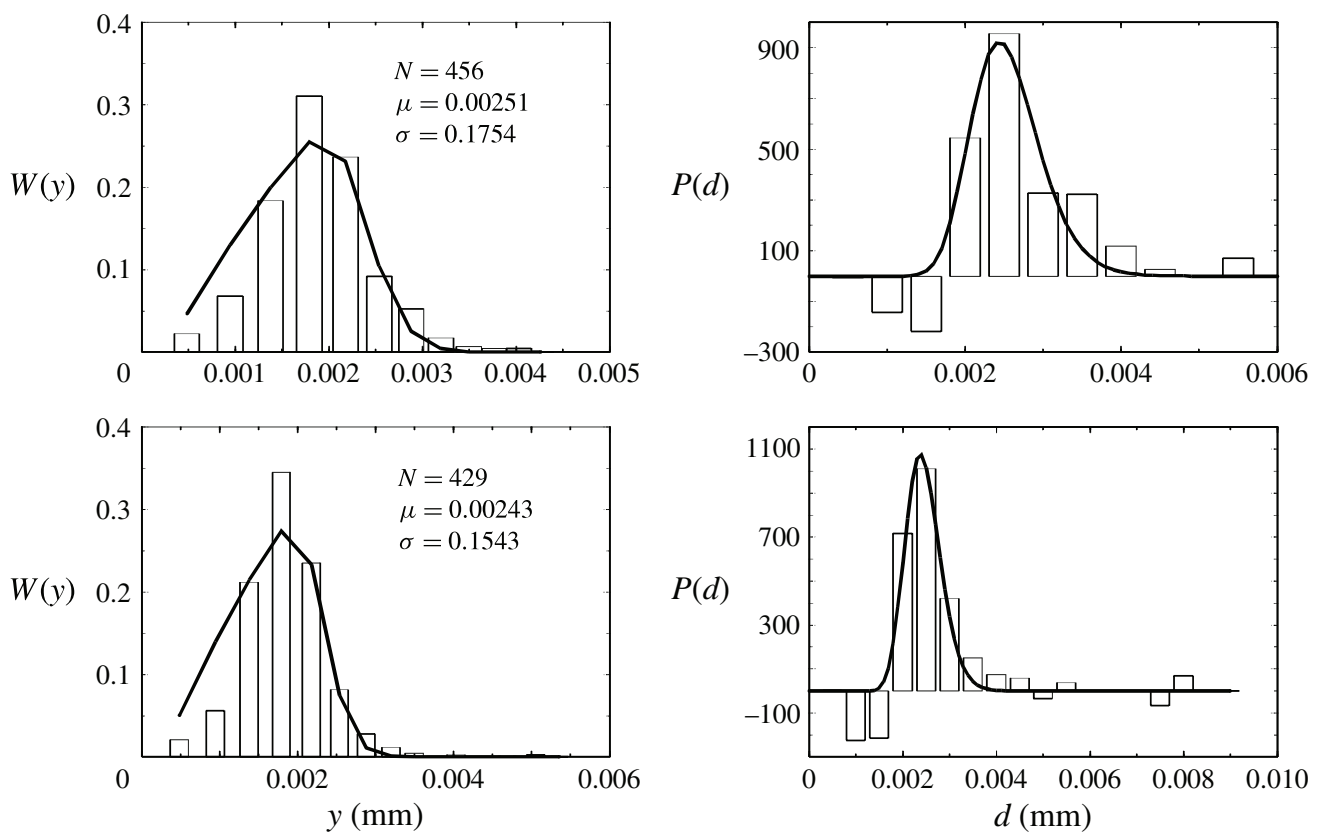

FIGURE 17. Bubble diameter distributions $P(d)$ versus chord length distribution $W(y)$ for two different flow conditions: comparison between a direct backward transformation, (A 11) (bars), and a least-square minimization assuming that the bubble distribution follows a lognormal law (A 14) (solid line).

For air-water flow at ambient conditions of pressure and temperature, the liquid properties are $\rho_{L}=1000 \mathrm{~kg} \mathrm{~m}^{-3}, v=10^{-6} \mathrm{~m}^{2} \mathrm{~s}^{-1}, \sigma=0.07 \mathrm{~N} \mathrm{~m}^{-1}$ and the acceleration due to gravity $\gamma$ is equal to $9.81 \mathrm{~m} \mathrm{~s}^{-2}$ so that the aspect ratio $E$ becomes the solution of

$$
E=\frac{1}{1+1283 d^{1.514} E^{1.009}} \quad \text { with } M o=2.68 \times 10^{-11} .
$$

The above equation can be fitted by $E=1-52.6 d$, for $d$ between 1 and $6 \mathrm{~mm}$. This simplified expression of $E$ is replaced by (A 7), (A 8) and (A 11) to (A 13) are used to calculate the p.d.f. of the bubble sizes.

Then different characteristic diameters can be calculated: $d_{10}$, the equivalent mean diameter; $d_{20}$, the averaged diameter weighted by the bubble surface $d^{2} f_{E}(d) ; d_{30}$, the averaged diameter weighted by the bubble volume $E d^{3}$ :

$$
\begin{gathered}
d_{10}=\int_{0}^{\infty} E^{1 / 3} d P(d) \mathrm{d} d, \\
d_{20}=\left[\int_{0}^{\infty} f_{E}(d) d^{2} P(d) \mathrm{d} d\right]^{1 / 2}, \\
d_{30}=\left[\int_{0}^{\infty} E d^{3} P(d) \mathrm{d} d\right]^{1 / 3}, \\
f_{E}(d)=\frac{1}{2}+\frac{E^{2}}{2 \sqrt{1-E^{2}}} \log \frac{1+\sqrt{1-E^{2}}}{E} .
\end{gathered}
$$



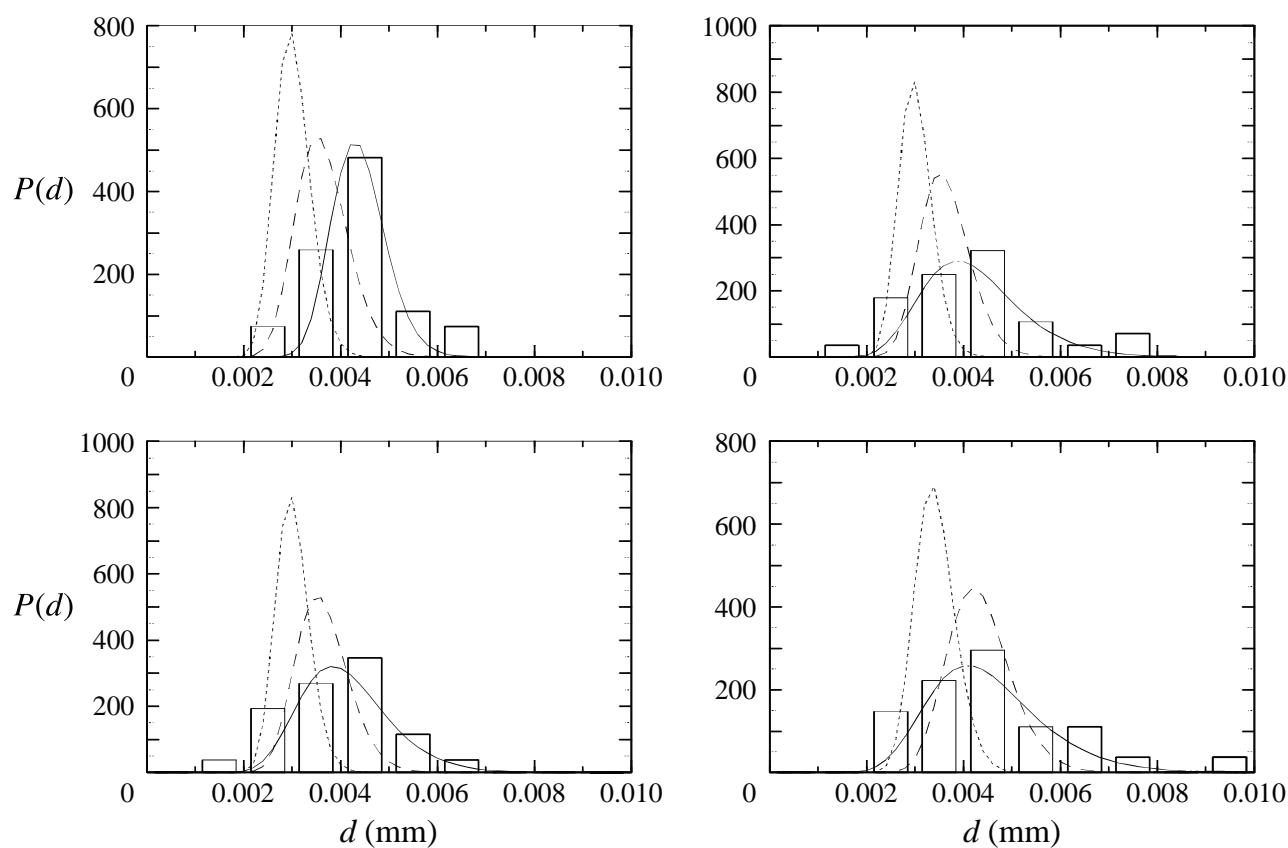

FIGURE 18. Bubble size distributions for four different runs: comparison between opticalfibre measurements assuming spherical (dotted lines) or ellipsoidal (dashed lines) bubbles and image processing measurements from high-speed video pictures (bars and solid lines).

The interfacial area per unit volume $A_{i}$ can be calculated from $d_{30}$ and $d_{20}$ and from the Sauter diameter $d_{32}$ as follows:

$$
d_{32}=\frac{d_{30}^{3}}{d_{20}^{2}}, \quad A_{i}=\frac{6 \alpha}{d_{32}},
$$

where $\alpha$ is the local gas fraction.

\section{A.3. Validation of the method}

High-speed video pictures of the flows were taken for four different runs in downward flow. The minor and major axes of the bubbles were measured from image processing. Then the bubble size distribution across the section was determined. In downward flow the bubble size is uniform across the section. Thus a good estimation of the bubble size distribution is obtained from a measurement at a single point of the cross-section. The bubble size distributions determined from the chord lengths measured by the optical-fibre probe are plotted in figure 18 .

The calculations were carried out for both spherical and ellipsoidal bubble shapes and the p.d.f.s were compared to those obtained from image processing. The average and standard deviation $\mu$ and $\hat{\sigma}$ are reported for downward flow conditions in table 4. The spherical shape assumption leads to bubble sizes much smaller than those determined by image processing whereas the ellipsoidal bubble shape assumption gives a closer agreement even if the sizes remain smaller and the p.d.f. narrower. It seems that the Wellek correlation for rising bubbles in still liquid underestimates the bubble deformation in turbulent flow. The comparison between the measured and calculated mean bubble diameter shows that the discrepancy is smaller than $20 \%$ for 


\begin{tabular}{|c|c|c|c|c|c|c|c|}
\hline \multicolumn{2}{|c|}{ Flow conditions } & \multicolumn{2}{|c|}{ Image processing } & \multicolumn{2}{|c|}{$\begin{array}{c}\text { Optical fibre } \\
\text { Spherical bubbles }\end{array}$} & \multicolumn{2}{|c|}{$\begin{array}{l}\text { Optical fibre } \\
\text { Ellipsoidal bubbles }\end{array}$} \\
\hline $\begin{array}{l}j_{L} \\
\left(\mathrm{~m} \mathrm{~s}^{-1}\right)\end{array}$ & $\begin{array}{c}j_{G} \\
\left(\mathrm{~m} \mathrm{~s}^{-1}\right)\end{array}$ & $\underset{(\mathrm{mm})}{\mu}$ & $\begin{array}{c}\hat{\sigma} \\
(\mathrm{mm})\end{array}$ & $\begin{array}{c}\mu \\
(\mathrm{mm})\end{array}$ & $\begin{array}{c}\hat{\sigma} \\
(\mathrm{mm})\end{array}$ & $\begin{array}{c}\mu \\
(\mathrm{mm})\end{array}$ & $\begin{array}{c}\hat{\sigma} \\
(\mathrm{mm})\end{array}$ \\
\hline 1.00 & 0.024 & 4.4 & 0.18 & 3.0 & 0.17 & 3.6 & 0.21 \\
\hline 1.00 & 0.040 & 4.1 & 0.34 & 3.0 & 0.16 & 3.6 & 0.20 \\
\hline 0.77 & 0.0185 & 4.0 & 0.32 & 3.0 & 0.16 & 3.6 & 0.21 \\
\hline 0.77 & 0.053 & 4.4 & 0.36 & 3.4 & 0.17 & 4.3 & 0.21 \\
\hline
\end{tabular}

TABLE 4. Log-normal law parameters in vertical downward flow for bubble sizes distributions determined by image processing and optical probe measurements.

all the cases. A better estimate of the bubble deformation in turbulent flow should be included in further development of the model. Another important parameter is the orientation of the minor axis of the bubbles. In this model, the bubbles were assumed to move with a velocity parallel to their minor axis and to the pipe axis. Even if the minor axis of the bubbles tends to move towards the direction of their instantaneous velocity (Ellingsen \& Risso 2001) it does not necessarily remain parallel to the pipe axis.

\section{REFERENCES}

Adoua, R., Legendre, D. \& Magnaudet, J. 2009 Reversal of the lift force on an oblate bubble in a weakly viscous linear shear flow. J. Fluid Mech. 628, 23-41.

Antal, S. P., Lahey, R. T. \& Flaherty, J. E. 1991 Analysis of phase distribution in fully laminar bubbly two-phase flow. Intl J. Multiphase Flow 17, 635-652.

Chahed, J., Colin, C. \& Masbernat, L. 2002 Turbulence and phase distribution in bubbly pipe flow under microgravity condition. Trans. ASME: J. Fluids Engng 124, 951-956.

Chahed, J. \& MASBERnAt, L. 1998 Effets de paroi sur la distribution de taux de vide dans les écoulements à bulles. C. R. Acad. Sci. IIb 326, 719-726.

Chahed, J., Roig, V. \& Masbernat, L. 2003 Eulerian-Eulerian two-fluid model for turbulent gas-liquid bubbly flows. Intl J. Multiphase Flow 29, 23-49.

Clark, N. N. \& TURTON, R. 1988 Chord length distribution related to bubble size distributions in multiphase flows. Intl J. Multiphase Flow 14, 413-424.

Clift, R., Grace, J. R. \& Weber, M. E. 1978 Bubbles, Drops and Particles. Academic.

Colin, C., FAbre, J. \& Dukler, A. E. 1991 Gas liquid flow at microgravity conditions. I. Dispersed bubble and slug flow. Intl J. Multiphase Flow 17, 533-544.

Colin, C., FABre, J. \& MCQuillen, J. B. 1996 Bubble and slug flow at microgravity conditions: state of knowledge and open questions. Chem. Engng Commun. 141-142, 155-173.

Colin, C. \& Legendre, D. 2001 Bubble distribution in turbulent shear flows: experiments and numerical simulations on single bubbles. In 4th International Conference on Multiphase Flows, New Orleans, USA (ed. S. Michaelides). CD Rom Publication.

Csanady, G. T. 1964 Turbulent diffusion of heavy particles in the atmosphere. J. Atmos. Sci. 21, 201-208.

Deutch, E. \& Simonin, O. 1991 Large eddy simulation applied to the modelling of particulate transpot coefficients in turbulent two-phase flows. In 8th Symposium on Turbulent Shear Flow, Technical University of Munich, Germany.

Drew, D. \& LAHEY, R. T. 1982 Phase distribution mechanisms in two-phase flow in a circular pipe. J. Fluid Mech. 117, 91-106.

DUINEVELD, P. 1994. Bouncing and coalescence of two bubbles in water. PhD thesis, University, Twente, Netherland. 
ELLINGSEN, K. \& Risso, F. 2001 On the rise of an ellipsoidal bubble in water: oscillatory paths and liquid-induced velocity. J. Fluid Mech. 440, 235-268.

EPPINGER, K. 1995 Etude du mouvement des bulles dans une turbulence homogène isotrope. Thesis, INP, Toulouse, France.

Fabre, J., Masbernat, L. \& Suzanne, C. 1983 Some remarks on the constitutive equations of stratified gas-liquid flow. In Multiphase Flow and Heat Transfer III (ed. T. N. Veziroglu \& A. E. Bergles). Fundamental, vol. A. pp. 41-57. Elsevier.

Farar, B., Samways, A. L. \& BruUn, H. H. 1995 A computer based technique for two-phase flow measurements. Meas. Sci. Technol. 6, 1528-1537.

Fujiwara, A., Minato, D. \& Hishida, K. 2004 Effect of bubble diameter on modification of turbulence in an upward pipe flow. Intl J. Heat Fluid Flow 25, 481-488.

GARnier, C., LANCE, M. \& MARié, J.-L. 2001 Measurement of local flow characteristics in buoyancy-driven bubbly flow at high void fraction. In 4th International Conference on Multiphase Flow, New Orleans, USA (ed. S. Michaelides). CD Rom Publication.

GrossetÊTE, C. 1995 Experimental investigation and preliminary numerical simulation of void development in a vertical cylindrical pipe. In 2nd International Conference on Multiphase Flow (ed. A. Serizawa, T. Fukano \& J. Bataille), Kyoto, Japan, Elsevier.

HASAN, Y. A., Schmidl, W. \& ORTIZ-Villafuerte, J. 1998 Investigation of the threedimensional two-phase flow structure in a bubbly pipe flow. Meas. Sci. Technol. 9, 309-326.

Hazaku, T., Takamasa, T. \& Hibiki, T. 2012 Characteristics of developing vertical bubbly flow under normal and microgravity conditions. Intl J. Multiphase Flow 38, 53-66.

HERRINGE, R. A. \& DAVIS, M. R. 1976 Structural development of gas-liquid mixture flows. J. Fluid Mech. 73, 97-123.

Hibiki, T., GodA, H., Kim, S., Ishit, M. \& Uhle, J. 2004 Structure of vertical downward bubbly flow. Intl J. Heat Mass Transfer 47, 1847-1862.

HinZE, J. O. 1959 Turbulence. McGraw-Hill.

Hosokawa, S., Suzuki, T. \& Tomiyama, A. 2010 Effects of bubbles on turbulence properties in a duct flow. Multiphase Sci. Technol. 22, 211-232.

Hosokawa, S. \& Tomiyama, A. 2004 Turbulence modification in gas-liquid and solid-liquid dispersed two-phase pipe flows. Intl J. Heat Fluid Flow 25, 489-498.

Hosokawa, S. \& TomiYama, A. 2009a Application of photobleaching molecular tagging velocimetry to turbulent bubbly flow in a square duct. Exp. Fluids 47, 745-754.

Hosokawa, S. \& TOMiYama, A. $2009 b$ Multi-fluid simulation of turbulent bubbly pipe flows. Chem. Engng Sci. 64, 5308-5318.

KAMP, A. 1996 Ecoulements turbulents à bulles dans une conduite en micropesanteur. Thesis, INP, Toulouse, France.

Kamp, A., Colin, C., Chesters, A. K. \& Fabre, J. 2001 Bubble coalescence in turbulent flow: a mechanistic model for turbulence induced coalescence applied to microgravity bubbly pipe flow. Intl J. Multiphase Flow 27 (8), 1363-1396.

KASHINSKY, O. N. \& RANDIN, V. V. 1999 Downward bubbly gas-liquid flow in a vertical pipe. Intl J. Multiphase Flow 25, 109-138.

LAhey, R. T. \& BonetTo, F. 1994 Analysis of phase distribution phenomena in microgravity environments. In 2nd Microgravity Fluid Physics Conference, Cleveland, Ohio, USA, pp. 193-201. NASA.

LAMB, H. 1932 Hydrodynamics. Dover.

LANCE, M. \& BATAiLle, J. 1991 Turbulence in the liquid phase of a uniform bubbly air-water flow. J. Fluid Mech. 225, 95-118.

LANCE, M. \& LOPEZ DE Bertodano, M. 1994 Phase distribution phenomena and wall effects in bubbly two-phase flows. In Multiphase Science and Technology: Two-phase Flow Fundamentals (ed. G. F. Hewitt, J. H. Kim, R. T. Lahey, J.-M. Delhaye \& N. Zuber), vol. 8. pp. 69-123. Begell House.

Larue de Tournemine, A. 2001 Etude expérimentale de l'effet du taux de vide en écoulement diphasique à bulles. Thesis, INP, Toulouse, France. 
LAUfer, J. 1954 The structure of turbulence in fully developped pipe flow. NACA Rep. 1174.

Legendre, D., Colin, C., Fabre, J. \& Magnaudet, J. 1999 Influence of gravity upon the bubble distribution in a turbulent pipe flow: comparison between numerical simulations and experimental data. J. Chim. Phys. 96, 951-957.

LiU, T. J. 1989 Experimental investigation of turbulence structure in two-phase bubbly flow. $\mathrm{PhD}$ thesis, Northwest University, Evanston, Illinois.

LiU, T. J. 1993 Bubble size and entrance length effects on void development in a vertical channel. Intl J. Multiphase Flow 19, 99-113.

LIU, T. J. 1998 The role of bubble size on liquid turbulent structure in two-phase bubbly flow. In 3rd International Conference on Multiphase Flow Lyon, France (ed. J. Bataille). CD Rom Publication.

LiU, T. J. \& BANKOFF, S. G. 1993a Structure of air-water bubbly flow in a vertical pipe-1. Liquid velocity and turbulence measurements. Intl J. Heat Mass Transfer 36, 1049-1060.

LiU, T. J. \& BANKofF, S. G. $1993 b$ Structure of air-water bubbly flow in a vertical pipe-2 Void fraction, bubble velocity and bubble size distribution. Intl J. Heat Mass Transfer 36, 1061-1072.

LiU, W. \& CLARK, N. N. 1995 Relationships between distributions of chord lengths and distributions of bubble sizes including their statistical parameters. Intl J. Multiphase Flow 21, $1073-1089$.

Lopez de Bertodano, M., Lahey, R. T. \& Jones, O. C. 1994 Phase distribution in bubbly two-phase flow in vertical ducts. Intl J. Multiphase Flow 20, 805-818.

Lucas, D., Krepper, E. \& Prasser, H.-M. 2007 Use of models for lift, wall and turbulent dispersion forces acting on bubbles for poly-disperse flows. Chem. Engng Sci. 62, 4146-4157.

Magnaudet, J., Rivero, M. \& Fabre, J. 1995 Accelerated flows past a rigid sphere or a spherical bubble. Part 1. Steady straining flow. J. Fluid Mech. 284, 97-135.

Marié, J.-L., Moursali, E. \& Tran-Cong, S. 1997 Similarity law and turbulence intensity profiles in a bubbly boundary layer at low void fraction. Intl J. Multiphase Flow 23, 227-247.

Mendelson, H. D. 1967 The prediction of bubble terminal velocities from wave theory. AIChE J. 13, 250-253.

Michiyoshi, I. \& Serizawa, A. 1986 Turbulence in two-phase bubble flow. Nucl. Engng Des. 95, 253-267.

Nakoryakov, V. E., Kashinsky, O. N., Burdukov, A. P. \& Odnoral, V. P. 1981 Local characteristics of upward gas-liquid flow. Intl J. Multiphase Flow 7, 63-81.

NAKoryakov, V. E., KAShinsky, O. N., RAndin, V. V. \& Timkin, L. S. 1996 Gas-liquid bubbly flow in vertical pipes. Trans. ASME: J. Fluids Engng 118, 377-382.

Powell, M. 1964 An efficient method for finding the minimum of a function with several variable without calculating derivatives. Comput. J. 7, 155-162.

Prasser, H.-M., Krepper, E. \& LuCAS, D. 2002 Evolution of the two-phase flow in a vertical tube: decomposition of gas fraction profiles according to bubble size classes using wire-mesh sensors. Intl J. Therm. Sci. 41, 17-28.

Riboux, G., Risso, F. \& LEgEndRe, D. 2010 Experimental characterization of the agitation generated by bubbles rising at high Reynolds number. J. Fluid Mech. 643, 509-539.

SAто, Y. \& Yамамото, K. 1987 Lagrangian measurements of fluid-particle motion in approximately homogeneous turbulence. J. Fluid Mech. 88, 63-72.

SCHLichting, H. 1968 Boundary-layer Theory. McGraw-Hill.

Serizawa, A. \& Kataoka, I. 1988 Phase distribution in two-phase flow. In Transient Phenomena in Multiphase Flow (ed. N. H. Afghan). pp. 179-225. Hemisphere.

Serizawa, A. \& KatAoKA, I. 1990 Turbulence suppression in bubbly two-phase flow. Nucl. Engng Des. 122, 1-16.

Serizawa, A., Kataoka, I. \& Michiyoshi, I. 1975 Turbulence structures of air-water bubbly flow -II. Local properties. Intl J. Multiphase Flow 2, 236-246. 
Shawkat, M. E., Ching, C. Y. \& Shoukri, M. 2008 Bubble and liquid turbulence characteristics of bubbly flow in a large diameter vertical pipe. Intl J. Multiphase Flow 34, 767-785.

Takamasa, T., Iguchi, T., Hazaku, T., Hibiki, T. \& IshiI, M. 2003 Interfacial area transport of bubbly flow under microgravity environment. Intl J. Multiphase Flow 29, 291-304.

TCHEN, C. M. 1947 Mean value and correlation problems connected with the motion of small particles suspended in a turbulent flow. PhD thesis, Technische Hogeschool, Delft, Netherlands.

Theofanous, T. G. \& Sullivan, J. 1982 Turbulence in two-phase dispersed flows. J. Fluid Mech 116, 343-362.

Tomiyama, A., TAmai, H., Zun, I. \& HosokaWA, S. 2002 Transverse migration of single bubbles in simple shear flows. Chem. Engng Sci. 57, 1849-1858.

WANG, S. K. 1985 Three-dimensional turbulence structure measurements in air-water two-phase flow. PhD Thesis, Nucl. Eng. Dept., Troy, New York.

WAng, S. K., Lee, S. J., Jones, O. C. \& LAhey, R. T. 1987 3-D turbulence structure and phase distribution measurements in bubbly two-phase flows. Intl J. Multiphase Flow 13, 327-343.

VAN DER WELle, R. 1985 Void fraction, bubble velocity and bubble size in two-phase flow. Intl J. Multiphase Flow 11, 317-345.

YeUnG, P. K. \& Pope, S. B. 1989 Lagrangian statistics from direct numerical simulation of isotropic turbulence. J. Fluid Mech. 207, 531-586.

ZUN, I. 1988 Transition from wall void peaking to core void peaking in turbulent bubbly flow. In Transient Phenomena in Multiphase Flow (ed. N. H. Afghan). Hemisphere.

Zun, I., Kljenak, I., Serizawa, A. \& Moze, S. 1993 Space time evolution of non homogeneous bubble distribution in upward flow. Intl J. Multiphase Flow 19, 151-172. 